\title{
IV. Frau und Öffentlichkeit
}

\section{A. Die Einbeziehung der Frau in die gewerbliche Wirtschaft}

1933-1939

\begin{abstract}
I. Die erste Phase 1933 bis 1936 - die Begrenzung der Frau auf ein Hausfrauen- und Mutterdasein
\end{abstract}

Schon vor 1933 hatten sich große Teile der NSDAP dafür ausgesprochen, die Frau aus Beruf und Universität ganz zu verbannen und sie auf ihren ,angestammten“ Platz als „verständnisvolle Begleiterin“ des Mannes und "Wahrerin des Blutes" zu verweisen.' Dieses Vorhaben entsprach der ideologischen Bewertung der Frau. Seiner Ausführung kamen zwei Umstände entgegen: Zum einen war die Diskussion um die Stellung der Frau im Berufsleben noch keineswegs abgeschlossen, und es gab immer noch genügend einflußreiche Stimmen, die sich gegen die wirtschaftliche und politische Emanzipation der Frau aussprachen, ${ }^{2}$ zum andern wurde der Rückzug der Frauen aus der Wirtschaft und die positive Haltung weiter Bevölkerungskreise dazu durch die Wirtschaftskrise erleichtert. ${ }^{3}$

Als Zielsetzung der ersten Phase wurde formuliert, ,die deutsche Frau aus der Erwerbswirtschaft überhaupt herauszunehmen und ausschließlich ihren Aufgaben innerhalb der Verbrauchswirtschaft als Ehefrau und Mutter wiederzuzuführen. ${ }^{4}{ }^{4}$ Um dieses Ziel zu erreichen, wurden verschiedene Maßnahmen ergriffen. Innerhalb des Staatsapparates entließ man zunächst die Beamtinnen, die „nicht die Gewähr dafür bieten, daß sie jederzeit rückhaltlos für den nationalen Staat eintreten", ${ }^{5}$ und die Doppelverdiener. Im übrigen beschränkte man sich auf propagandistische Appelle, ${ }^{6}$ bei Neueinstellungen oder vor die Alternative gestellt, einen Beamten oder eine Beamtin zu entlassen, hatte - bei gleicher Eignung - der männliche Bewerber allerdings den absoluten Vorrang. Nur für den Bereich der Jugendarbeit und der Mädchenbildung wurden weibliche Kräfte als notwendig angesehen. ${ }^{\text {? }}$

\footnotetext{
1 Veeh, S. 64 f. Die folgende Untersuchung über Frau und Wirtschaft beruht auf meiner unveröffentlichten Staatsexamensarbeit vom Februar 1977. Die später zu diesem Thema erschienenen Arbeiten von Dörte Winkler, Frauenarbeit im „Dritten Reich“. Hamburg 1977 und von Stefan Bajohr, Die Hälfte der Fabrik. Marburg 1978 bringen für den untersuchten Zeitraum nichts wesentlich Neues.

2 Vgl. Kapitel I. I.

${ }^{3}$ Mason, Arbeiterklasse, S. 5 I.

${ }^{4}$ Hans Land: Die Bekämpfung der Schwarzarbeit, des Doppelverdienertums und der Frauenarbeit im Rahmen der deutschen Konjunkturpolitik. Diss. Marburg 1935, S.45.

${ }^{5}$ RGBI. I 933, Teil I (7.4. 1933), S. I 75, \$4. Gertrud Bäumer: Zum „Status“ der deutschen Frau. In: Die Frau 44 (1936/37), S. 643, (zit.: Bäumer, Status).

${ }^{6}$ Mason, Arbeiterklasse, S. 53 und S. 53 Anm. 30.

7 VB (Berl. Ausg.) I 3. IO. 1933 (ministerielle Stellungnahme). Veeh, S. 66 (zur Bedeutung der Frau für die Mädchenbildung).
} 
Auch auf Industrie und Handel wurde kein offizieller Zwang zur Entlassung von Frauen und Mädchen ausgeübt, wohl aber inoffizieller Druck. ${ }^{8}$ Teil dieser Versuche, bei der Durchsetzung der Parteiziele nachzuhelfen, war die kurze, aber heftige Kampagne gegen verheiratete Frauen als Doppelverdiener, die aber nicht den erhofften Erfolg hatte, ${ }^{9}$ zumal sich Rudolf $\mathrm{Heß}$ wegen der vielfältigen damit zusammenhängenden Probleme genötigt sah, eine Verfügung zu erlassen, die Parteigenossen (Pgs.) und Parteistellen verbot, von sich aus Regelungen in dieser Frage zu treffen. ${ }^{10}$

Die Forderung nach Ersatz der Frauen- durch Männerarbeit bestand zwar auch noch weiterhin sowohl aus ideologischen wie auch aus wirtschaftlichen Gründen, ${ }^{11}$ es stellte sich aber heraus, daß an eine generelle Beseitigung der Frauenarbeit gar nicht zu denken war ${ }^{12}$ und auch bei der Ersetzung bestimmte Probleme bedacht werden mußten: So war die Frau z. T. die einzige Ernährerin der Familie, die bei ihrem Ausscheiden aus dem Berufsleben der staatlichen Unterstützung zur Last fiele, bzw. nicht jede Frau konnte das Ideal der Hausfrau und Mutter erreichen. ${ }^{13}$ Zudem würden Teile der Kaufkraft vernichtet, und auch für den Betrieb wäre die Einstellung von Männern anstelle von Frauen nicht immer wünschenswert, da er dann das Lohnniveau wesentlich erhöhen müßte, was möglicherweise auch die angestrebte Mengenkonjunktur gefährden könnte. ${ }^{14}$ Außerdem stellte sich die Frage, ob Frauen- und Männerarbeit überhaupt austauschbar waren, denn das relative Anwachsen der Frauenberufstätigkeit ging nur im ganz geringen Maße darauf zurück, daß Männer ersetzt wurden. Die Anteilsvergrößerung resultierte zum größten Teil aus Entstehung und Ausbau neuer Industrien und aus der Rationalisierung in typisch männlichen Arbeitsbereichen. ${ }^{15}$ Ein absolutes Zurückdrängen der Frauenarbeit war also in der ersten Phase weder möglich, noch - nach wirtschaftlichen Gesichtspunkten - wünschenswert, eine partielle Umschichtung aber unerläßlich. Als erste Umstrukturierungsmaßnahme zur Entlastung des Arbeitsmarkts wurde die Einstellung von Hausgehilfinnen gefördert. Hausangestellte wurden von der Zahlung der Arbeitslosenversicherung ,befreit', und wenn sie im Haushalt des Arbeitgebers lebten, fielen sie bei der Einkommensteuer des Hausherrn unter die Kinderermäßigung. ${ }^{16}$ Innerhalb eines Jahres wurden so 100000 Arbeitsplätze in der Industrie von Frauen geräumt. ${ }^{17}$

Das Gesetz zur Förderung der Eheschließungen vom x . 6. I 933 stellte beim Prozeß der Her-

\footnotetext{
${ }^{8}$ Mason, Frauen, S. I 36.

9 Ebenda, S. 139.

10 Soziale Praxis Nr. 42 (1933), Sp. I 398 f.

1 Ebenda, Sp. 1237.

12 Land, S.62.

13 Solange es nicht möglich ist, daß jede Frau heiratet, „gibt uns der Staat das Recht einen Beruf zu wählen“. VB (Süddt. Ausg.), r 8. November 1934, Jugendbeilage.

14 Land, S. 60-62.

15 Kaiser, S. III.

${ }_{16}$ RGBl. I 933, Teil I (I 2.5. I933), S. 265, Art. I (x). RGBl. 1933, Teil I (I.6. I 933 ), S. 326, Abschn. IV Art. I.

17 Theodor Splettstößer: Die Bekämpfung der männlichen Arbeitslosigkeit durch Herausnahme von Frauen aus dem Erwerbsleben unter besonderer Berücksichtigung der Problematik des Familienlastenausgleichs. Diss. Greifswald I 935, S. 27 (weitere Zahlen liegen mir nicht vor).
} 
auslösung der Frauen aus dem gewerblichen Bereich die wichtigste Maßnahme dar, es verfolgte aber darüber hinaus auch noch weitergehende politische Ziele. „Das Gesetz zur Förderung der Eheschließungen stellt nicht nur ein Arbeitsbeschaffungsprogramm dar, nicht nur eine sogenannte Initialzündung, nicht nur ein künstliches Mittel zur Verminderung der Arbeitslosigkeit, sondern es handelt sich hier um eine grundlegende arbeitsmarktpolitische und bevölkerungspolitische Umschichtung unserer deutschen Frauen und damit um eine organische und dauernde Verminderung der Arbeitslosigkeit. Allein diese Maßnahme wird zu einem weiteren starken Rückgang der Arbeitslosigkeit und zu einer Belebung in allen Zweigen der deutschen Wirtschaft führen." 18

Nach dem Gesetz waren zur Förderung von Eheschließungen Ehestandsdarlehen bis zu ro00 RM möglich, wenn die zukünftige Ehefrau zwischen dem 1.6. 1931 und dem 31.5. 1933 mindestens 6 Monate lang Arbeitnehmerin gewesen war und sich verpflichtete, ihre Tätigkeit aufzugeben und nicht eher wieder aufzunehmen, ,als der künftige Ehemann Einkünfte ... von mehr als 125 Reichsmark monatlich bezieht und das Ehestandsdarlehen nicht restlos getilgt ist. "19 Das Darlehen wurde allerdings nicht in bar ausgezahlt, sondern in Form von (zweckgebundenen) Bedarfsdeckungsscheinen vergeben, mit denen das Paar Möbel und Hausgeräte erwerben konnte, ${ }^{20}$ was wohl nicht nur zur Gründung des Hausstandes notwendig war, sondern gleichzeitig die Konjunktur in bestimmten Bereichen beleben sollte. Zur Finanzierung der Darlehen wurde die bis dahin übliche Ledigensteuer durch eine Ehestandshilfe ersetzt, die zwischen 2 und 5\% des Einkommens lag. ${ }^{21}$ Die erste Durchführungsverordnung vom 20. Juni 1933 präzisierte das Gesetz noch. So konnte das Darlehen nicht gewährt werden, „wenn nach der politischen Einstellung eines der beiden Ehegatten anzunehmen ist, daß er sich nicht jederzeit rückhaltlos für den nationalen Staat einsetzt". ${ }^{22}$ Ferner wurde bestimmt, daß für jedes lebendgeborene Kind $25 \%$ des Darlehens erlassen wurde und daß nach der Geburt eines Kindes auf Antrag die Rückzahlung für $\mathbf{I} 2$ Monate unterbrochen werden konnte. ${ }^{23}$ Privatbetriebe, die die Tendenz des Gesetzes durch freiwillige Heiratsbeihilfen unterstützten, erhielten steuerliche Begünstigungen. ${ }^{24}$

Schon 1934 wurden die Auswirkungen des Gesetzes als voller Erfolg gefeiert. Bis Januar 1934 waren I 83000 Darlehen gewährt und damit I 83000 Arbeitsplätze für Männer gewonnen worden, hinzu kamen in der Möbel- und Hausgeräteindustrie noch I 50000-1 80000 neue Arbeitsplätze, d.h. der Arbeitsmarkt war um 300000-350000 Arbeitslose entlastet worden. ${ }^{25}$ Bis zum 3 I. I 2. I 934 war die Arbeitslosenziffer durch die Ehestandsmaßnahmen - nach offiziellen Angaben - um 500000 gesunken, und der Finanzbedarf der Arbeitslosenhilfe hatte sich um etwa 250 Mill. Reichsmark verringert. ${ }^{26}$

\footnotetext{
18 Staatssekretär Reinhardt. In: Der Kongreß zu Nürnberg, S. 72.

${ }^{19}$ RGBl. 1933, Teil I (1.6. I933), S. 326 f., \ I. $\quad{ }^{20}$ Ebenda, $\ 3$.

${ }^{21}$ Ebenda, $\$ 4$. Rühle, I. Bd., S. 230. Nach Broszat, S. I 17 wurden die ersten Ehestandsdarlehen noch aus Mitteln des Reinhardtprogramms gefördert.

22 RGBl. 1933, Teil I (20.6. 1933), I. DVO S. 377.

${ }^{23}$ Ebenda. Auf die Wiedergabe der anderen Durchführungsverordnungen wird verzichtet, da sie keine wesentlichen Änderungen brachten.

24 Land, S. 63.

${ }^{25}$ Meier-Benneckenstein, 2. Bd., S. 172 f. $\quad{ }^{26}$ Ursachen und Folgen, 9. Bd., S. 703.
} 
Für die Ehewilligen, die mit Hilfe des Darlehens einen Hausstand gründen wollten, wirkte sich der Ansturm auf die Gelder allerdings ziemlich negativ aus. Betrug die durchschnittliche Höhe des Ehestandsdarlehen im August und September noch 730 RM, so sanken die Beträge im Februar auf eine Höhe von 560 RM ab. Bewilligungsbescheide mußten laut Anweisung von Anfang 1934 bis zum Ende des Rechnungsjahres am 3 I. 3 . 1934 hinausgezögert werden. ${ }^{27}$ Ehestandsdarlehen und Rückzahlungsmodi hatten nicht nur die direkte Förderung der Geburtenrate zum Ziel, sondern man versprach sich davon auch, daß sie indirekt dazu beitrügen, die Geburtenzahl zu steigern, denn wenn die Fabrikarbeit für kinderlose oder kinderarme Frauen, die aus Not arbeiteten, akzeptabel erschien, so hatte man doch festgestellt, daß sich die gewerbliche Arbeit hemmend auf die Geburtenrate auswirkte. Die „Zufluchtstätte der Unfruchtbaren“ barg die Gefahr in sich, zur „Lehrstätte der Unfruchtbarkeit“" zu werden, dazu kamen dann noch die Schädigungen durch den Beruf selbst. ${ }^{28}$

Der in der Propaganda so stark hervorgehobene Erfolg, durch die Rückführung der Frau in die Familie die Arbeitslosigkeit verringert zu haben, war allerdings nur relativ. Zwar sank der Anteil der Frauen in der Industrie gegenüber den männlichen Industriearbeitern von 29, 3\% (1933) auf 24,7\%,(1 936), ${ }^{29}$ doch hieß dies nicht, daß die Zahl der Industriearbeiterinnen abgenommen hatte. ${ }^{30}$ Bezogen auf alle krankenversicherungspflichtigen Arbeitnehmer nahm die Zahl der beschäftigten weiblichen Arbeitskräfte zwischen 1933 und 1936 kontinuierlich zu, sie stieg von 4580000 (I 933) auf 5338000 (I936). Mit anderen Worten: waren 1933 nur $80,5 \%$ der Frauen, die überhaupt dem Arbeitsmarkt zur Verfügung standen, beschäftigt, so erhöhte sich 1936 der Anteil auf 92,4\%. Parallel dazu vergrößerte sich auch die Zahl der weiblichen Arbeiter und Angestellten, die vom Arbeitsamt vermittelt wurden. ${ }^{31}$

\section{Die zweite Phase I936-I 939}

\section{a) Die Frau im Zeichen des Vierjabresplans}

Der Versuch die Frau auf ihre Funktion als Hausfrau und Mutter zu begrenzen, wurde mit der zunehmenden Erschöpfung der Arbeitskraftreserven 1935/36 aufgegeben. Die ideologischen Forderungen und Zielsetzungen hatten sich in diesem Bereich der aktuellen wirtschaftlichen Situation anzupassen. ${ }^{1}$ Die Rückgewinnung der Frau für den Arbeitsprozeß war aber auch langfristig von besonderer Bedeutung, denn „die Frau wird im Ernstfall im großen Umfang die Arbeit in den Fabriken leisten müssen. Sie muß also dafür vorbereitet werden. Auch hier müssen sich die sozialen Bestrebungen, die Frau aus den Betrieben zu lö-

\footnotetext{
27 Reichsgesundheitsblatt 1934, S. 36 I f.

28 Rott u.a., S. 69 f.

29 Statistisches Jahrbuch für das Deutsche Reich 1937, S. 344.

30 Geht man davon aus, daß die Zahl der männlichen Industriearbeiter stieg, so kann prozentual gesehen bei gleichbleibender Zahl der weiblichen Industriearbeiter der Anteil der Frauen sinken.

31 Statistisches Jahrbuch für das Deutsche Reich I937, S.613. I933 waren es I 054000, I936 I 505000 .

1 Vormschlag, S. 206. Honigberger, S. 73.
} 
sen, den militärischen Notwendigkeiten unterordnen . . . Feststeht, daß auf vielen Gebieten die Frau eine glänzende Facharbeiterin werden kann und auch bereits jetzt schon dementsprechende Arbeit tut". ${ }^{2}$

Schon $1935 / 36$ deutete sich der Umschwung in der NS-Frauenwarte, dem parteiamtlichen Frauenblatt, an. Die Beiträge des dritten Jahrgangs I $934 / 35$ wiesen noch auf die vielfältigen Gefahren für die berufstätige Frau hin, Gefahren, die die Stenotypistin ebenso trafen wie die Tabakarbeiterin und die Frau im Krankendienst. ${ }^{3}$ Im vierten Jahrgang wurden Vertreterinnen dieser Berufe als Beispiel herausgestellt für die besonderen Fähigkeiten der Frau zu diesen Arbeiten und für ihre innere Berufung dazu. ${ }^{4}$ Auch an der Handhabung der Regelung für Ehestandsdarlehen läßt sich der Trend zur Rückführung der Frau in die Berufswelt erkennen. Schon im Juli 1936 konnte der Finanzminister aufgrund der vierten Durchführungsverordnung ausnahmsweise Frauenarbeit gestatten, auch wenn das Darlehen noch nicht zurückgezahlt oder die Familie in einer besonderen Notlage war. ${ }^{5}$ Im November r 937 wurde die Frauenarbeit dann - trotz Ehestandsdarlehen - freigegeben, lediglich der Tilgungsbetrag für das Darlehen erhöhte sich von I \% auf $3 \% .^{6}$

Nach der Verkündung des Vierjahresplans stellte sich das Problem, „ob nicht Möglichkeiten zur Auffüllung des männlichen Arbeitsmarktes durch den Einsatz weiblicher Arbeitskräfte vorhanden sind?"7 Diese Frage konnte eindeutig bejaht werden. „Neben [!] der groBen Aufgabe, die die Frau als Mutter für ein Volk hat, steht ihre Bedeutung als ,Arbeitsreserve', welche besonders in Notzeiten in Anspruch zu nehmen ist." ${ }^{8}$ Wenn auch das Heranziehen der Reservearmee mit gewissen ,Gefahren“ verbunden war (Vernachlässigung der hausfraulichen Ausbildung, mögliche Senkung der Geburtenrate ${ }^{9}$ ), so erschien doch der Verzicht auf „die größte Reserve, über die Deutschland verfügte“" ${ }^{10}$, nicht möglich. $1938 /$ 39 stand „die Frage der Frauenberufstätigkeit . . nicht mehr zur Diskussion in Anbetracht der Tatsache, daß jede einzelne Arbeitskraft dringend zum Aufbau unserer Volkswirtschaft gebraucht wird“. ${ }^{11}$ Der erhöhte Arbeitseinsatz hatte auch - nach parteioffizieller Ansicht für die Frau selbst erhöhte Bedeutung, er hatte endgültig die falschen Vermutungen über die nationalsozialistische Auffassung zum Schweigen gebracht: Die Entwicklung der „letzten Jahre“ war gekennzeichnet durch die steigende Teilnahme der Frauen am Berufsleben und „durch die steigende Wertung und Anerkennung der fraulichen Mitarbeit auf allen Ar-

\footnotetext{
2 Wortprotokoll der 5 . Tagung der Reichsarbeitskammer vom 24. November I 936, zit. nach Mason, Arbeiterklasse. S. $185 \mathrm{f}$.

${ }^{3}$ FW 3 (r934/35), Heft Is, S. 466.

${ }^{4} \mathrm{FW}_{4}$ (I935/36), Heft II, S. 333.

${ }^{5}$ RGBl. I 936, Teil I (28.7. I 936), S. $576, \$$ I.

${ }^{6} \mathrm{RGBl}$. I 937, Teil I(3. I I. 1937), S. I I 59, \2; danach galt das Ehestandsdarlehen nun auch für landwirtschaftliche Betriebe.

7 Marie Elisabeth Lüders: Zu viel Arbeit - zu wenig Hände! In: Die Frau 44 (I 936/37), S. 494, (zit.: Lüders, Zu viel Arbeit).

${ }^{8}$ Ursula Becker: Die Entwicklung des Frauenerwerbs seit der Jahrhundertwende. Diss. Breslau 1937, S. 6.

$9 \mathrm{VB}$ (Süddt. Ausg.) 28. I 2. 1937.

${ }^{0}$ Friedrich Syrup: Neue Maßnahmen zur Regelung des Arbeitseinsatzes. In: Der Vierjahresplan 2 (1938), S. I 44, (zit.: Syrup, Maßnahmen).

"FW 7 (1938/39), Heft 16, S. 490.
} 
beitsgebieten“. ${ }^{12}$ Trotzdem konnte der Mangel an Arbeitskräften bis 1939 nicht behoben werden. ${ }^{13}$ Die absolute Zahl der weiblichen Beschäftigten stieg zwar weiterhin kontinuierlich an, zwischen I 936 und 1939 erhöhte sich der Prozentsatz der beschäftigten weiblichen Arbeitnehmerinnen von $92,4 \%$ auf etwa $96,4 \%{ }^{14}$ und auch der Anteil der weiblichen Industriearbeiter erhöhte sich (bezogen auf 100 Industriearbeiter) im selben Zeitraum von 24,7 auf 27,0 (Juni 1939), ${ }^{15}$ aber die Steigerung vollzog sich relativ langsam, so daß erst I $94 \mathrm{I}$ die Zahl der weiblichen Berufstätigen von 1928 überschritten wurde. ${ }^{16}$ Die Wiedereingliederung der Frau in die gewerbliche Wirtschaft reichte aber nicht aus, um die Ziele des Vierjahresplans zu erreichen. Parallel zur Steigerung der Frauenberufsarbeit mußte auch versucht werden, die Frauen in bestimmte Berufe und Industriezweige zu lenken, die rüstungspolitisch wichtig waren. Teil der Berufslenkung war das Pflichtjahr für Frauen, das nur beim Eintritt in bestimmte Berufe abgeleistet werden mußte. ${ }^{17}$

Wieviel Planung und Lenkung des Berufseinsatzes ein Erfolg war, läßt sich ohne eine Berufsspartenstatistik nicht feststellen. Man kann nur konstatieren, daß die Zahl der beschäftigten weiblichen Arbeitsbuchpflichtigen zwischen 1933 und 1938 in Industrie und Handwerk prozentual stärker zugenommen hat als in anderen Bereichen. ${ }^{18}$ Nicht klar ist, ob sich diese Zunahme hauptsächlich auf rüstungswichtige Betriebe und Industriezweige konzentrierte. ${ }^{19}$ Es gibt verschiedene Gründe dafür, daß die Wiedereinbeziehung der Frau ins Erwerbsleben trotz einer gewissen Zunahme der weiblichen Arbeitnehmer nicht den gewünschten Erfolg hatte. Weibliche Angestellte, die von der Heim- und Herd-Ideologie offensichtlich besonders stark angesprochen wurden, kehrten nach ihrer Heirat im allgemeinen nicht mehr in den Beruf zurück, ${ }^{20}$ aber auch bei anderen Gruppen von Frauen ist diese Tendenz zu beobachten. Sie hatten kein Interesse mehr an der Berufstätigkeit. Die Ehe war zum Teil Flucht vor der Monotonie der Arbeit, die gerade für Frauen so adäquat erschien, und durch die Verbesserung der allgemeinen finanziellen Lage war die Frau nicht mehr so stark auf den eigenen Verdienst angewiesen. ${ }^{21}$ Selbst für Arbeiterfamilien, die in der Regel mehr Kinder hatten als Familien anderer Schichten, war durch Arbeitskräftemangel und die verschiedenen Kinderbeihilfen eine Verbesserung ihrer Lage eingetreten. ${ }^{22}$ Ein weiterer Grund ist die Zunahme der Geburtenrate, denn bei relativ gesichertem Einkommen werden

${ }^{12} \mathrm{FW} 7($ (1938/39), Heft 22, S. 693 .

${ }^{13}$ Ebenda, S.694.

${ }_{14}$ Statistisches Jahrbuch für das Deutsche Reich 1937 , S. 334; 1938, S. 358 ; 1939/40, S. 372.

${ }_{15}$ Statistisches Jahrbuch für das Deutsche Reich 1937, S. 344; 1939/40, S. 386.

${ }^{16}$ Statistisches Handbuch für das Deutsche Reich S. 474.

17 Syrup, Sozialpolitik, S. 450.

${ }_{18}$ Statistisches Jahrbuch für das Deutsche Reich 1939/40, S. 379.

19 Statistische Angaben liegen mir dazu leider nicht vor. Nach Ilse Buresch-Riebe: Frauenleistung im Kriege. Berlin I 94 I, S. 29 war der Frauenanteil in den kriegswichtigen Industrien schon vor dem Krieg ziemlich hoch, es läßt sich aber nicht feststellen, ob es sich bei dieser Aussage um Tatsachen oder um Propaganda handelt.

${ }^{20}$ Mason, Frauen, S. $x 65$. Nach A. M. Hanne: Das Frauenamt der DAF. In: Jahrbuch der Reichsfrauenführung I 937, S. 49 waren nur $7 \%$ aller weiblichen Angestellten verheiratet.

${ }^{21}$ Mason, Frauen, S. $\mathrm{x} 59 \mathrm{f}$.

${ }^{22}$ Ebenda, S. $16 \mathrm{r}$. 
Frauen mit Säuglingen und Kleinkindern nur selten geneigt gewesen sein, noch einen Beruf auszuüben.

Auch die Löhne, die den Frauen gezahlt wurden, waren kaum ein Anreiz, einer gewerblichen Tätigkeit nachzugehen. Löhne und Gehälter für Arbeitnehmerinnen lagen, trotz der Bedeutung, die die Frau für die Wirtschaft zunehmend gewann, weit unter den entsprechenden Tarifen für Männer, ${ }^{23}$ wenn auch zeitweise das Einkommen der Frauen stärker stieg als das der Männer. ${ }^{24} \mathrm{Daß}$ sich an diesem Lohngefälle grundsätzlich nichts änderte auch nicht im Kriegsfall, als die Frauenarbeit noch wichtiger wurde - gewährleistete ein Erlaß des Reichsarbeitsministers: Bei notwendiger Ergänzung der Lohnsätze durch spezielle Frauentarife war davon auszugehen, daß Frauen höchstens $75 \%$ des Männerlohns erhielten. ${ }^{25}$ Gerechtfertigt wurde diese Lohnstaffelung mit soziologischen Gegebenheiten, deren Aufhebung auch die Aufhebung der gesellschaftlichen Ordnung implizieren würde und die deshalb aufs schärfste abzulehnen sei. ${ }^{26}$ Darüber hinaus sollte den Frauen eingeredet werden, die hauswirtschaftliche Aufklärung durch staatliche Stellen käme einer indirekten Lohnerhöhung gleich. ${ }^{27}$ Der weiterhin bestehende Mangel an Arbeitskräften machte neben einer Leistungssteigerung die Einbeziehung verheirateter Frauen notwendig, eine Maßnahme, die allerdings nur vorübergehenden Charakter haben sollte, ${ }^{28}$ denn trotz der Notwendigkeit Arbeitskräfte zu finden, sollten die in der ersten Phase aufgestellten Maximen nicht total aufgegeben werden. „Neben dem Vierjahresplan müssen vor allem jene Aufgaben weitergeführt werden, deren Ziel es ist, das deutsche Volk gesünder und sein Leben angenehmer zu machen." 29 Dazu zählte der Schutz der werktätigen Frau, der von der sozialen Betriebsarbeiterin und dem DAF-Frauenamt gewährt wurde. Als konkrete Schutzmaßnahme sind die Arbeitszeitverordnung vom 30.4. 1938 und die Richtlinien für den Mutterschutz zu werten. In der Arbeitszeitverordnung wurde bestimmt, daß selbst bei Ausnahmeregelungen, die der Reichsarbeitsminister erlassen konnte, die Arbeitszeit nicht mehr als Io Stunden betragen durfte. ${ }^{30}$ Die Richtlinien für den erweiterten Mutterschutz von 1937 verpflichteten die Schwangere, innerhalb der Schutzzeit die Arbeit niederzulegen. Der Betrieb gewährte ihr in dieser Zeit den Unterschiedsbetrag zwischen Wochengeld und Wochenver-

${ }^{23}$ Statistisches Jahrbuch für das Deutsche Reich 1937, S. 316 ; 1939/40, S. 35 2; Statistisches Handbuch für das Deutsche Reich, S. 469.

${ }^{24}$ Die stärkere Zunahme der Frauenlöhne trifft für das erste Vierteljahr $1937 \mathrm{zu}$. Vgl.: Bericht des Statistischen Reichsamts über die Entwicklung der tatsächlichen Arbeitsverdienste im I. Vierteljahr 1937. Auszug bei Mason, Arbeiterklasse, S. 3 r $2 \mathrm{f}$.

${ }^{25}$ Erlaß des Reichsarbeitsministers an die Treuhänder der Arbeit vom 23. August 1939. In: Mason, Arbeiterklasse, S. $104 \mathrm{I}$.

${ }^{26}$ Elisabeth Oehlandt: Deutsche Industriearbeiterinnenlöhne 1928-1935. Rostock 1937, S. $102 \mathrm{f}$.

27 Hanne, S. 54.

${ }^{28} \mathrm{FW}_{7}(1938 / 39)$, Heft 22, S.694. Nach Mason, Frauen S. I 58 stieg die Zahl der verheirateten erwerbstätigen Frauen sehr stark an, zwischen 1933 und 1939 stieg die Zahl der verheirateten Frauen um I I ,5\%, die Zahl der erwerbstätigen verheirateten Frauen um 24,8\%. Beim Rückgriff auf verheiratete Frauen, die bisher nicht im Berufsleben standen, war man allerdings sehr vorsichtig. Selbst im September 1939 erschien es noch nicht tunlich, diese Frauen durch Dienstverpflichtung in den Produktionsprozeß einzugliedern. Vgl. dazu Gersdorff, S. 296 f. (Verfügung des Reichsarbeitsministers vom 7.9. 1939).

${ }^{29} \mathrm{FW}_{7}$ (1 938/39), Heft 9, S. 28 r.

${ }^{30}$ RGBl. 1938, Teil I (30.4. 1938), S. 450, \17(3). 
dienst. ${ }^{31}$ Die Tatsache, daß diese Regelung erst 1937 getroffen wurde, trotz der ständigen Beteuerungen, wie wichtig und schützenswert die zukünftige Mutter sei, läßt sich nur daraus erklären, daß bis 1936 der Anteil der erwerbstätigen Frauen möglichst gesenkt werden sollte; nun aber, da die Frauenarbeit gefördert werden mußte, war es notwendig, die Schwangere, die im Berufsleben stand, stärker zu schützen, wollte man auf Kinder nicht verzichten. Selbst für den Kriegsfall, wo „in weitestem Umfange Frauen in Wirtschaft und Verwaltung eingesetzt werden müssen, um wehrfähige Männer für den Kampf mit der Waffe freizustellen ... muß die Frauenarbeit dort ihre Grenze finden, wo sie den Lebensquell der Nation bedrohen würde“".32 Als äußeres Zeichen dafür, daß man den „Willen zum Kind" nicht aufgegeben hatte, sind wohl auch die kleineren bereits beschriebenen Vergünstigungen zu werten, die hauptsächlich kinderreiche Familien betrafen.

Auch bei der Leistungseinschätzung der Frau läßt sich eine gewisse Ideologiekonstanz feststellen. So sollten nach den „Richtlinien für die Beschäftigung von Frauen im Mobfall“" Frauen möglichst nicht mit Aufgaben betraut werden, die ,typisch männliche‘ Eigenschaften erforderten, wie Geistesgegenwart, Entschlußkraft, schnelles Handeln und besonderes technisches Verständnis. ${ }^{33}$ Zudem hatten Frauen, so dringend sie auch gebraucht wurden, nur so geringe Aufstiegschancen im Beruf, ${ }^{34} \mathrm{da}$ sich Frau Scholtz-Klink, die Reichsfrauenführerin, zu einem Schreiben an den Stellvertreter des Führers veranlaßt sah, in dem sie die zunehmende Tendenz beklagte, ,hochbegabte, leistungsfähige Frauen in ihrer Arbeit nicht weiterkommen zu lassen, ihre Leistungen, nur weil sie von einer Frau kommen, nicht zu fördern“, und dringend eine grundsätzliche Klärung verlangte. ${ }^{35}$

Diese grundsätzliche Klärung war, auch wenn die Reichsfrauenführerin dies nicht zur Kenntnis genommen hatte, längst erfolgt. So sehr die nationalsozialistische Frauenideologie mit der industriellen Frauenerwerbstätigkeit kollidierte, so erfüllte sie in einem ganz kleinen Bereich doch eine wichtige Funktion; denn um Frauen aus hochqualifizierten und öffentlich wirksamen Positionen zu entfernen, bediente man sich der Ideologie als Legitimation. In diesem Teilbereich des Berufslebens war es auch am einfachsten, gegen die Frauenarbeit vorzugehen. Die Zahl der betroffenen Frauen war gering, und bei den Beamtinnen brauchte der NS-Staat ohnehin nur noch zu sanktionieren und zu legitimieren, was bereits in der Weimarer Republik an praktischer Benachteiligung gang und gäbe war. ${ }^{36}$ Besonders die Akademikerinnen, gegen die traditionell bis heute männliche Vorurteile und die Angst vor dem Konkurrenzdruck existieren, fielen den Maßnahmen zum Opfer. So wurden bereits 1933 die meisten weiblichen Direktoren an den Schulen durch Männer ersetzt, ${ }^{37}$ in der Schulverwaltung gab es überhaupt keine Frauen mehr. ${ }^{38}$ Selbst Ärztinnen, die doch das Ide-

\footnotetext{
${ }_{31}$ Hildegard Backendorf: Mutterschutzgesetz. Berlin/Leipzig/Wien 1944, S. I s f.

32 Reichsarbeitsminister am 16.9. 1938, zit. nach Gersdorff, S. 286.

${ }^{33}$ Gersdorff, S. 286 f.

34 Karl Dietrich Bracher: Die deutsche Diktatur. Köln/Berlin I 970³, S. 368.

${ }^{35}$ Brief vom 24. I. 1938, zit. nach Gersdorff, S. 285.

36 Winkler, S. so.

37 Die Deutsche Kämpferin 3 (1935/36), S. 78. Nach dem I. S. 1933 gab es nur noch 5 Direktorinnen in Preußen. Ludwig Wülker: Die Neubesetzung der Direktorenstellen an den deutschen öffentlichen höheren Mädchenbildungsanstalten. In: Deutsche Mädchenbildung 1935, S. 46.

${ }^{38}$ Die Deutsche Kämpferin 1 (1933/34), S. 123.
} 
al der sozial tätigen Frau geradezu verkörperten, sahen sich vor fast unüberwindliche Schwierigkeiten gestellt. ${ }^{39}$ Für die Juristin gab es ab I 936 ein weitreichendes Berufsverbot: Durch eine persönliche Entscheidung Hitlers waren ihr der Staatsdienst und das Amt des Notars verwehrt, ${ }^{40}$ selbst als Beisitzerin bei Erbgesundheitsgerichten ${ }^{41}$ oder für Prozesse vor den neuerrichteten Jugendkammern der Gerichte war sie nicht zugelassen. ${ }^{42}$ Lediglich in einzelnen Ministerien hatten noch einige Frauen eine hohe Position inne. Allerdings handelte es sich hier wohl um besonders qualifizierte Frauen, die nicht ohne weiteres ersetzt werden konnten und deren Tätigkeit in der Öffentlichkeit kaum bemerkt wurde. ${ }^{43}$

Betrachtet man die Gesamtentwicklung, wird noch einmal deutlich: Die Frau war im NSStaat keine Persönlichkeit, kein Individuum, mit dem Recht zu selbständigen und eigenverantwortlichen Entscheidungen, sie eignete sich höchstens als Ersatz in Notzeiten. Die Frau war lediglich Mittel zu einem bestimmten, veränderbaren Zweck. Neben ihrer zentralen Funktion, möglichst viele erbgesunde Kinder in die Welt zu setzen, wurden - je nach Bedarf - noch andere Aufgaben an sie herangetragen, die sie zu erfüllen hatte. Im wirtschaftlichen Bereich wurde der Mittelcharakter besonders deutlich: Waren Arbeitsplätze knapp, wurde die Frau aufgefordert, sich den traditionellen Familienpflichten zu widmen, waren Arbeitskräfte knapp, wurde von ihr verlangt, zusätzlich einer Erwerbstätigkeit nachzugehen. ${ }^{44}$

\section{b) Die propagandistische Absicherung der zweiten Phase}

Die Ziele der zweiten Phase, aufgestellt in der Denkschrift zum Vierjahresplan, konnten nur verwirklicht werden, wenn sie vom Volk mitgetragen wurden und wenn genügend Arbeitskräfte für die Rüstungsproduktion zur Verfügung standen. Die Massen dementsprechend zu beeinflussen, darin „liegt die gewaltige Aufgabe der Bewegung mit ihrer Propaganda, Menschenführung und Menschenbeeinflussung". ${ }^{45}$

Für die Propaganda, die sich an die Frauen richtete, bedeutete das, daß sie vor die Aufgabe

39 Winkler, S. S 1. Es gab kaum noch die Möglichkeit, das obligatorische Assistenzjahr zu machen und die neue Zulassungsordnung der Krankenkassen erschwerte den Frauen die Aufnahme ihres Berufes. Nach Die Deutsche Kämpferin I (I 933/34), S. I 24 erwog die männliche Ärzteschaft sogar einen Entzug der Approbation für Frauen.

${ }^{40}$ Meier-Scherling: Die Benachteiligung der Juristin zwischen 1933 und I945. In: Deutsche Richterzeitung 1975, S. Ir.

41 Ebenda, S. I 2.

42 Die Frau 45 (1937/38), S. 5 I.

${ }^{43}$ Geht man davon aus, daß es regelmäßig ausgewiesen ist, wenn es sich um Frauen handelt, so ergibt sich nach: Die neuen Männer in den Reichs- und Länderministerien. Berlin 1933 folgendes Bild: Reich: Reichsminister des Innern, Abt. Wissenschaft, Bildung und Schule, Referentin Frau Siber (neben anderen männl. Referenten), Reichs-Wirtschaftsministerium, Abt. Währungsfragen, Referentin Frau Mende (neben männl. Referenten), Reichsarbeitsministerium, Abt. Arbeitsrecht, Arbeitsgerichtsbarkeit und Arbeitsschutz, Frau Lüders. Sachsen: Ministerium für Volksbildung Dr. phil. M. Dyck, Referentin für das höhere Schulwesen und für berufliche Schulen. Mecklenburg-Schwerin: Frau Petersen als Referentin im Ministerium für Unterricht, Kunst, geistliche und Medizinalangelegenheiten.

${ }^{4}$ Das ist allerdings kein typisch nationalsozialistischer Mechanismus, ähnliches spielt sich ja auch in der Bundesrepublik ab, wobei die Versuche, derartige Vorgänge zu legitimieren, allerdings grundsätzlich anders sind.

${ }^{45}$ Rede Görings in der ersten Sitzung des Reichsverteidigungsrates am r 8. November I 938. In: Mason, Arbeiterklasse, S. 909 . 
gestellt war, die Rückführung der Frau ins Berufsleben zu unterstützen und zu rechtfertigen. Gleichzeitig konnte und sollte aber das Ideal der ersten Phase - die Frau als Hausfrau und Mutter - nicht aufgegeben werden. Da sowohl die Aufrüstung (mit dem Ziel der Expansion) als auch die Idee der Mutterschaft wesentliche Bestandteile der nationalsozialistischen Ideologie waren, von denen keiner aufgegeben werden konnte, mußte in der Propaganda eine Lösung gefunden werden, die beiden Aspekte miteinander zu vermitteln, obwohl sie unvereinbar waren und sich gegenseitig ausschlossen. Ein Hilfsmittel bei der Bewältigung dieser Aufgabe war das Ideal der Volksgemeinschaft. Im Namen der Volksgemeinschaft war ja schon in der ersten Phase die absolute Unterordnung des Privatinteresses unter das Gesamtwohl gefordert worden, und daran konnte man jetzt anknüpfen. „Es gibt nur ein Interesse: das deutsche Volk und seine Zukunft, dem beugen sich alle." ${ }^{46}$ Im Hinblick auf das ,große Ziel“, das in Angriff genommen wurde, gab es keine Frauenfrage mehr, „es gibt nur eine einzige Frage jedes Deutschen: Wie hole ich das Beste aus mir heraus, damit mein Volk, damit Deutschland wieder so stark, so anständig, so gesund und so gläubig wird, daß es vor Gott und vor sich selbst bestehen kann. " ${ }^{47}$ Aus dem Gesamtinteresse resultierte für die Frau die staatspolitische Pflicht, in einer Zeit, in der jeder einzelne im Arbeitsprozeß gebraucht wurde, ihre Arbeitskraft in den Dienst des Volkes zu stellen. Jede Diskussion über diesen Schritt hatte hinter den ökonomischen Notwendigkeiten zurückzustehen. $^{48}$

Aber trotz der unbestreitbaren wirtschaftlichen Erfordernisse wurde die Berufstätigkeit der Frau nicht zum neuen Ideal der zweiten Phase erhoben, vielmehr wurde betont, daß die Frauenerwerbsarbeit eigentlich nur Ersatz sein konnte, denn ,über allem im Leben der Frau steht die Mutterschaft. . . So gewiß aber die Mutterschaft die höchste und beglückendste Aufgabe der Frau ist, so gewiß ist sie natürlich nicht die einzige." ${ }^{49}$ Gerade die Frauen, die auf eine Eheschließung verzichten mußten, leisteten in ihrem Beruf Hervorragendes ${ }^{50}$ und seien ,auf den Gebieten, die ihrer Natur entsprechen, voll anzuerkennen. “51 Diese Aussagen zeigen noch einmal das Dilemma, vor dem diejenigen standen, die aufgerufen waren, die Frau ins Berufsleben zurückzuführen. Die Frau sollte wieder berufstätig werden und mußte auch die nötige Anerkennung für die erbrachte Leistung erhalten, aber da das Muttertum doch ihr eigentliches Schicksal war, ${ }^{\text {s2 }}$ konnte die Berufstätigkeit nur vorläufigen Charakter haben.

Ein weiteres Zugeständnis an das einmal aufgestellte Frauenideal ist in der Betonung der ,naturgemäßen Arbeit' zu sehen, wenn sich auch der Inhalt dessen, was frauengemäß war, seit der ersten Phase geändert hatte. Selbst naturwissenschaftliche und technische Berufe

${ }^{46}$ Rede Görings auf der 6. Jahrestagung der DAF am ro. September 1938 (Auszug). In: Mason, Arbeiterklasse, S.677.

47 Scholtz-Klink In: FW 7 (1938/39), Heft 16, S. 489.

${ }^{48} \mathrm{FW} \mathrm{6}$ (1937/38), Heft 16, Umschlagseite - 489. Zur Notwendigkeit der Frauenarbeit: „Je mehr der Arbeitermangel in Deutschland wächst, desto mehr muß sich das Augenmerk zwangsläufig der Vermehrung der Frauenarbeit zuwenden." Soziale Praxis NF 47, (I 938 ), Sp. 1094.

${ }^{49}$ Scholtz-Klink: Frau und Beruf. In: Der Schulungsbrief 4 (I 937), Heft 3, S. xo I, (zit.: Scholtz-Klink, Frau).

50 Ebenda.

${ }^{51}$ H. Schemm (Gauleiter) in: Der Schulungsbrief 7 (1940), Heft 7-9, S. 106.

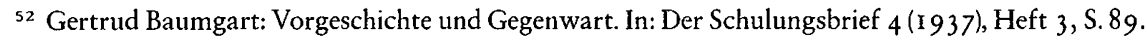


galten für die Frau nun als angemessen, ${ }^{53}$ da gerade in diesen Sparten Arbeitskräfte gebraucht wurden. Die frühere Begrenzung auf hauswirtschaftliche und soziale Tätigkeiten wurde offiziell abgelehnt, und man entdeckte ,spezielle frauliche Fähigkeiten' wieder, wie Zuverlässigkeit, Anpassungsfähigkeit und Geschicklichkeit. Aber obwohl diese ,natürlichen' Fähigkeiten der Frau in verschiedenen Berufen eingesetzt werden konnten, und trotz der betonten Hochachtung vor der erwerbstätigen Frau ${ }^{54}$ war doch nur in Ehe und Mutterschaft die höchste Erfüllung für die Frau zu finden, und nur die Frauen, die den Beruf mit der Kraft der seelischen Mutterschaft ausübten, konnten „neben die deutschen Mütter als Mitträgerinnen der Nation und Mitgestalterinnen des Volkes“ gestellt werden. ${ }^{55}$.

Die Bedeutung der Frau für die Familie wurde durch die Aufwertung der Hausfrau nochmals unterstrichen. Göring betonte in seiner Rede zum Vierjahresplan, wie wichtig das richtige Verhalten der Hausfrau für das Gelingen des Plans sei, ${ }^{56}$ und da 60-80\% des Volksvermögens durch ihre Hand gingen, wurde sie sogar zur „Treuhänderin des Volksvermögens“ erklärt. ${ }^{57}$

Parallel zur ideologischen Stilisierung der Hausfrau verlief der Versuch, auch diesen Bevölkerungskreis für den Vierjahresplan zu aktivieren. So wurde die Frau aufgerufen, sparsam und pflegerisch mit den Gegenständen des täglichen Bedarfs umzugehen, die Ernährungsweise an die Jahreszeit und ihre Erzeugnisse anzupassen und die Lebensmittel rationell zu verwenden. Insgesamt sollten die Bedürfnisse des täglichen Lebens soweit wie möglich ohne exportierte Waren befriedigt werden, damit zumindest eine relative Autarkie gewährleistet war. ${ }^{58}$ Auch die zunehmende Verbrauchslenkung wurde unter diesem Gesichtspunkt betrieben. ${ }^{59}$ Die Frauenzeitschriften veröffentlichten Rezepte zur rationellen Resteverwertung, Haltbarmachung von Lebensmitteln und für die Zubereitung verschiedener Produkte, deren Verzehr von staatlicher Seite aus gefördert werden sollte. ${ }^{60}$ Richtschnur für die gesamte Hausarbeit waren - wie in anderen Bereichen auch - die Belange der Volksgemeinschaft. Die Hausfrau mußte sich daran messen lassen, wie weit sie es verstand, ihre Haushaltsführung an den sogenannten Lebensnotwendigkeiten des Volkes auszurichten. ${ }^{61}$

Die Propagandisten waren Gefangene ihrer eigenen Propaganda geworden, denn trotz der Versuche, der berufstätigen Frau einen neuen Wert zuzuerkennen, weil sie dringend gebraucht wurde, trotz der Beteuerung, daß die Frau nichts von ihrem weiblichen Wesen verliere, wenn sie einem artgemäßen Beruf nachgehe, stand doch immer noch die Mutter an der Spitze der sozialen Rangskala. Die Geburten galten als die Siege der Frau, und nur in ih-

\footnotetext{
${ }^{53}$ Vormschlag, S. 204. FW S (1937/38), Heft 19, S.601.

${ }^{54}$ Scholtz-Klink, Frau, S. IOI.

${ }^{55}$ Scholtz-Klink, in: FW 7 (1938/39), Heft r6, S. 493.

${ }^{56}$ Berndt, S. 2 I 4.

${ }^{57}$ Eggener, S. 48.

${ }^{58}$ Else Vorwerck: Die Hausfrau im Dienste der Volkswirtschaft. o. O., o. J., S. 3-14, (zit.: Vorwerck, Hausfrau).

${ }^{59}$ Nach Zeitschriften-Dienst, Berlin, Nr. 42 war das Wort Verbrauchslenkung zu vermeiden.

${ }^{60}$ Ebenda. Es sollte nicht von Fischgestank geredet werden, sondern man sollte den gesundheitlichen Vorteil von Fisch hervorheben.

${ }^{61}$ Vorwerck, Hausfrau, S. 4.
} 
nen erfüllte sich ihr Wert. ${ }^{62}$ Geachtet wurde - notgedrungen - die berufstätige Frau, aber „heilig soll uns sein jede Mutter guten Blutes. " ${ }^{63}$ Der Versuch, das neue Frauenideal mit den Erfordernissen der Rüstungskonjunktur in Einklang zu bringen, war damit letztlich gescheitert. Die Propaganda unterstützte eigentlich eher die Tendenz, nur aus materieller Not in der Industrie tätig zu werden, anstatt diese Haltung zu beseitigen. Die Mutterschaftsideologie hatte sich verselbständigt, d.h. sie hatte keinen Bezug mehr zu den realen Notwendigkeiten, ja sie widersprach ihnen sogar.

\section{Die berufliche Organisierung der Frau}

Getreu der NS-Frauenideologie, die behauptete, daß Mann und Frau grundsätzlich verschieden seien, erfolgte die berufliche Organisierung der Erwerbstätigen. In nahezu jeder Berufsorganisation gab es eine Sonderabteilung, die sich unter weiblicher Führung speziell um frauliche Belange zu kümmern hatte. Die bedeutendste Abteilung dieser Art war das im August 1934 geschaffene Frauenamt der DAF, das bereits Ende 1935 rund 7 Millionen Mitglieder „betreute“. ${ }^{1}$ Der hohe Organisationsgrad bot optimale Voraussetzungen zur Kontrolle und Lenkung der betroffenen Frauen. Offiziell hatte das DAF-Frauenamt die Aufgabe, die Interessen der Frau zu wahren und zu vertreten. Dabei war es den organisierten Frauen keineswegs möglich, diese Interessen selbst zu definieren oder zu artikulieren. Welche Belange die weibliche „Gefolgschaft“ hatte, bestimmte das Frauenamt, ihre Vertretung und Durchsetzung besorgte die vom Frauenamt ernannte Vertrauensfrau, die als einzige Vertreterin aller fraulichen Belange im Betrieb anerkannt war. ${ }^{2}$

Ausgangspunkt und Zielrichtung wurden durch die neue Bewertung der Frau am Arbeitsplatz festgelegt. „Die Frau wird nicht mehr gewertet als ,siegberechtigter Gegner' des Mannes, sondern als Arbeitskameradin, die in erster Linie Frau ist und deren Arbeitsweise, ihre Leistungen und Voraussetzungen unter denen diese geschieht, dem weiblichen Wesen angepaßt sein müssen. ${ }^{{ }_{3}}$ Folgerichtig erstreckte sich die Hauptaufgabe der Vertrauensfrau auch auf Mutterschutz im weitesten Sinne. Durch ihre Arbeit sollte die Gebärfähigkeit der Frau im Arbeitsprozeß geschützt werden, außerdem versuchte man, „der erwerbstätigen Frau auch diejenigen Kenntnisse zu verschaffen, die zur Erfüllung mütterlicher und hausfraulicher Aufgabe notwendig sind. “ ${ }^{4}$

In Betrieben, die mehr als 250 Frauen beschäftigten, übernahm eine sogenannte „Soziale Betriebsarbeiterin“ die Aufgaben der Vertrauensfrau. ${ }^{5}$ Während die übrigen Vertrauens-

\footnotetext{
62 „Weil die Geburten meine Siege sind. In ihnen ist mein Wert als Frau erfüllt." Geleit der deutschen Frau. In: Der Schulungsbrief 7 ( I 940), Heft 7-9, S. ro3.

${ }^{63} \mathrm{FW} 7$ (1938/39), Heft 6, S. 166.

1 DAF, Abt. Frauen BA, Slg. Schumacher/230, Blatt I.

2 Nationalsozialistische Parteikorrespondenz Folge 71 , Blatt 8, 26.3. 1935.

${ }_{3}$ BA, Slg. Schumacher/2 30, Blatt x. Um die Gebärfähigkeit der Frau zu erhalten, forderte die DAF auch gleiche Löhne für Männer und Frauen, denn so glaubte man verhindern zu können, daß Frauen wegen der Lohnersparnis für schwere Arbeiten eingesetzt würden.

${ }^{4}$ BA, Slg. Schumacher/2 30, Blatt I. $\quad{ }^{5}$ Eggener, S. 70.
} 
frauen offenbar nur eine kurze Schulung mitmachten und nebenamtlich tätig waren, mußte die Soziale Betriebsarbeiterin neben der Kenntnis der betrieblichen Arbeit eine sozial- und arbeitspädagogische Ausbildung vorweisen, die sie befähigen sollte, „dem Betriebsführer und dem Vertrauensrat in der Sorge um das Wohl der Gefolgschaft helfend zur Seite“ zu stehen. ${ }^{6}$

Obwohl immer wieder betont wurde, daß die gesamte Arbeit des DAF-Frauenamts für die Frau geleistet wurde, läßt sich doch unschwer erkennen, daß der „Einsatz“ der Vertrauensfrau im Grunde nur die Interessen des Staates wahren sollte. Neben dem Schutz der Gebärfähigkeit, die dem Regime so ungeheuer wichtig war, stand als letztes Ziel hinter den Bemühungen die „Bildung einer wirklichen Volks- und Leistungsgemeinschaft aller Deutschen “, d.h. die Bildung eines nationalsozialistischen Bewußtseins auch unter den Frauen. Unter diesem Blickwinkel muß auch die Tatsache gesehen werden, daß für größere Betriebe Frauen mit einer speziellen Ausbildung als Vertrauensfrau herangezogen wurden. Nach eigener Einschätzung der Machthaber war nämlich im Fabrikbetrieb der Gemeinschaftsgedanke am wenigsten durchgesetzt. ${ }^{8}$ Die Gesinnung der Frau auch in diesem Bereich auf nationalsozialistische Vorstellungen auszurichten erforderte daher besondere Qualifikationen.

Außer dieser ganz allgemeinen Unterordnung unter die Prinzipien der Volksgemeinschaft, die als langfristiges Erziehungsprogramm zu sehen ist, war das Frauenamt vor eine praktische, kurzfristig zu lösende Aufgabe gestellt. Bereits sehr früh hatten die zuständigen Stellen die Konsequenz aus den Versäumnissen des Ersten Weltkrieges gezogen und erkannt, daß auch die Frau systematisch auf den Kriegsdienst vorbereitet werden mußte. Die Mobilisierung der weiblichen Arbeitskräfte war der erste Schritt auf diesem Weg. Nach der Eingliederung in den Arbeitsprozeß sollte das Frauenamt dann unter Berücksichtigung des „wesensgemäßen Einsatzes“ der Frau und ihrer „, besonderen biologischen Aufgabe“ erstens für eine Leistungssteigerung in der Frauenarbeit sorgen ${ }^{9}$ und zweitens auch die Frau an eine ganz bestimmte Arbeitsdisziplin und Arbeitsmoral heranführen.

\section{B. Die Frau und ihre Beziehung zur Partei}

\section{Mitgliedschaft}

Auch wenn nach der ältesten gefundenen Mitgliedsliste der Frauenanteil in der NSDAP zunächst sehr hoch war, ${ }^{1}$ so ist dieses Verhältnis doch keineswegs als repräsentativ anzusehen. Im Verlauf der Parteientwicklung traten in der Hauptsache Männer in die Partei ein, so daß

\footnotetext{
- BA, Slg. Schumacher/2 30, Blatt 2.

7 NSK, Folge $7 x$, Blatt 8.

${ }^{8}$ Soziale Betriebsarbeit in der NS-Gau-Frauenschaft. In: FW 3 (1934/35), S. 4 I .

9 Buresch-Riebe, S. 37.

${ }^{1}$ Franz-Willing, S. 129.
} 
die Frauen bald auch zahlenmäßig keine Rolle mehr spielten. Da die Statistiken zur Mitgliedschaft im allgemeinen nicht nach Geschlechtern unterscheiden, muß die vom Reichsorganisationsleiter der NSDAP herausgegebene offizielle Parteistatistik Ausgangspunkt der nachfolgenden Überlegungen sein, auch wenn sie nur den Stand von 1935 (ohne Saarland) angibt. Für die spätere Zeit (bis 1939) fehlen derartige Unterlagen offensichtlich. Nach dieser Statistik waren am I. I. I935 von den 2493000 Parteimitgliedern I 36197 Frauen, ${ }^{2}$ das entsprach $5,5 \%$ der Parteigenossen. ${ }^{3}$ Vor der Machtergreifung waren bereits $47,0 \%$ der weiblichen Pgs. in die Partei eingetreten, allerdings nur 5,6\% vor dem I 4.9. I 930 , die restlichen $4 \mathrm{I}, 4 \%$ zwischen dem I 5.9. I 930 und dem 30. x. I $933 .{ }^{4}$ Für die Frau gewann die NSDAP also erst zu Beginn der 3 oer Jahre an Bedeutung, so wie sich umgekehrt auch die Partei erst in dieser Zeit näher mit der Frauenfrage befaßte.

Erstaunlicherweise waren es besonders die im allgemeinen als unpolitisch apostrophierten Hausfrauen, die sich zur NSDAP hingezogen fühlten; sie bildeten die größte Gruppe der weiblichen Parteimitglieder. Von I 36 I 97 Parteigenossinnen waren $64627(=47,4 \%$ ) Hausfrauen. ${ }^{5}$ 5 2,6\% von ihnen traten bereits vor dem I. 2. 1933 in die Partei ein, während im Reichsdurchschnitt, bezogen auf alle Parteimitglieder, nur $34 \%$ bis zu diesem Termin beigetreten waren. ${ }^{6}$ Am I. I . 1935 waren 0,7\% aller deutschen Hausfrauen Mitglieder der NSDAP, aber nur $0,5 \%$ der gesamten weiblichen Bevölkerung. ${ }^{7}$ Wie jede Partei bestand auch die NSDAP nicht nur aus einsatzfreudigen Mitgliedern. Nur 27,0\% der Parteigenossinnen beteiligten sich aktiv an der Parteiarbeit. ${ }^{8}$ Gemessen an der Zahl aller Parteimitglieder ist die Anzahl der Frauen sehr niedrig, gemessen am Frauenanteil der Bevölkerung $(\varsigma 2,2 \%)$ sind die Frauen ganz eindeutig unterrepräsentiert. ${ }^{9}$ Aber die Parteimitgliedschaft allein sagt noch wenig aus, denn auch ohne Parteibuch konnte sich die Frau aktiv für das Regime einsetzen, wie Geschichte und Entwicklung der NS-Frauenorganisationen zeigen.

\section{Wahlanalyse}

Die Frage nach dem Wahlverhalten der Frauen ist für die Weimarer Republik schwer zu beantworten, da die Wahlstatistiken nur zum ganz geringen Teil nach Geschlechtern differenzieren. ${ }^{1}$ Die folgenden Aussagen stecken also nur den Rahmen ab. Für die Stadt Köln, wo

\footnotetext{
2 Parteistatistik, S. I 2. ${ }^{3}$ Ebenda, S. 3 I. ${ }^{4}$ Ebenda, S. 13.

${ }^{5}$ Ebenda, S. I 3 1. ${ }^{6}$ Ebenda, S.74.

7 Ebenda, S. 40. Möglicherweise ist die überproportionale Vertretung der Hausfrau darauf zurückzuführen, daß es sich nicht um „Nur-Hausfrauen“ handelte, sondern um sogenannte mithelfende Familienangehörige ohne eigene Berufsausbildung. Es ist denkbar, daß sich diese Frauen, die trotz ihrer Mitarbeit ja keiner Erwerbsarbeit im eigentlichen Sinne nachgingen, selbst als Hausfrauen einstuften. Ein Eintreten in die Partei wegen der scheinbaren Aufwertung der Hausarbeit anzunehmen oder die Aufgabe der Berufsarbeit nach dem Parteieintritt zu vermuten, dürfte doch etwas zu weit gehen.

${ }^{8}$ Parteistatistik, S. 2 I $2 . \quad 9$ Ebenda, S. 40.

1 Die getrennte Erfassung ist gesichert für 6,9\% bei den Wahlen I924, 5,7\% bei den Wahlen I 928 , $20,6 \%$ bei den Wahlen I930, 16,8\% bei den Wahlen 1932. Heinrich Zurkuhlen: Wie wählen die Frauen? in: Die Tat 24 (1932), S. 545.
} 
eine Differenzierung erfolgte, stellt sich das Verhältnis von Männer- und Frauenstimmen für die NSDAP folgendermaßen dar: Auf 100 gültige Stimmen entfielen für die NSDAP ${ }^{2}$

\begin{tabular}{lrrr} 
Jahr & Männer & Frauen & zusam \\
\hline I 928 & 2,2 & I, 4 & I, 8 \\
I930 & I9,8 & I 5,5 & I 7,6 \\
1932 April & 29,9 & 24,3 & 27,1 \\
I932 Juli & 26,4 & 22,8 & 24,6 \\
1932 Nov. & 2 I, & 19,3 & 20,4
\end{tabular}

In einer Ưbersicht, die 1932 publiziert wurde, stellt sich das Wahlverhalten der Frauen bei den Reichstagswahlen 1924-I 930 folgendermaßen dar: ${ }^{3}$

Auf je roo männliche Wähler kommen weibliche Wähler.

\begin{tabular}{|c|c|c|c|c|}
\hline $\begin{array}{l}\text { Reichstags- } \\
\text { wahlen vom }\end{array}$ & $\begin{array}{l}4 \cdot 5 \\
1924\end{array}$ & $\begin{array}{l}7.12 . \\
1924\end{array}$ & $\begin{array}{l}20.5 . \\
1928\end{array}$ & $\begin{array}{l}14 \cdot 9 . \\
\text { I } 930\end{array}$ \\
\hline Bayerische Volkspartei & $\mathrm{I} 68,8$ & $x 55,7$ & I 57,0 & I 80,2 \\
\hline Zentrum & 144,5 & 143,0 & I 57,5 & \\
\hline Christl.-soz. Volksdienst & - & - & 130,0 & 222,0 \\
\hline Deutschnationale & $x \times 4,3$ & I I 5,0 & I 34,6 & 152,7 \\
\hline Volksrechtspartei & - & - & $\operatorname{x} 18,8$ & - \\
\hline Deutsche Volkspartei & $\operatorname{III}, 2$ & I I I ,9 & I I 3,7 & 136,5 \\
\hline Landbund & $\mathrm{I} O \mathrm{I}, 3$ & 99,2 & 89,5 & 97,9 \\
\hline Völkische & 95.6 & 83,0 & I I I ,4 & - \\
\hline Nationalsozialisten & & & 73,0 & 96,5 \\
\hline Wirtschaftspartei & 95,6 & 91,0 & 96,0 & 107,0 \\
\hline Deutsche Staatspartei & 94,9 & 94,2 & $102, \mathrm{I}$ & 109,0 \\
\hline Sozialdemokraten & 93,6 & 88,0 & 98,5 & 109,1 \\
\hline Unabhäng. Sozialdemokraten & 79,0 & 63,2 & 83,5 & - \\
\hline Deutsche Bauernpartei & 75,5 & 90,5 & 74,5 & - \\
\hline $\begin{array}{l}\text { Deutschhannoveraner } \\
\text { (einschl. Konserv. Volkspartei) }\end{array}$ & 73,6 & $88, \mathrm{I}$ & - & I I 4,8 \\
\hline Kommunisten & 69,0 & 66,3 & 78,5 & 82,6 \\
\hline
\end{tabular}

${ }^{2}$ Max Schneider: Frauen an der Wahlurne. In: Die Gesellschaft 10 (I933), S. 74.

${ }^{3}$ Zurkuhlen, S. 546. Die Aufstellung ist wegen methodischer und terminologischer Unklarheiten (z. B. Parteibezeichnungen) nur mit Einschränkungen brauchbar. 
Die Anzahl der Frauenstimmen für die NSDAP bleibt, wie aus den Zahlen zu ersehen ist, hinter den Männerstimmen zurück. Berücksichtigt man noch, daß die durchschnittliche Wahlbeteiligung bei Männern größer war als bei Frauen, ${ }^{4}$ wird der Abstand von Männerund Frauenstimmen für die NSDAP noch größer. Die Frauen gingen in ihrem Votum für Hitler und seine Partei nicht voran, wie die Zahlen zeigen, sondern sie zogen nur langsam nach. ${ }^{5}$ Das Übergewicht der männlichen Stimmen für die NSDAP kann auch noch anders verdeutlicht werden: „Rechnet man das Ergebnis einer Reichstagswahl unter ausschließlicher Berücksichtigung der männlichen Wählerstimmen in Mandate um, so ergeben sich in der Tat nicht unwesentliche Verschiebungen in der Fraktionsstärke . . . namentlich zugunsten der Kommunisten und der Nationalsozialisten." "Die Mehrzahl der weiblichen Wählerstimmen kam ganz eindeutig den konservativ christlichen Parteien zugute, ${ }^{7}$ während die radikalen Parteien das Nachsehen hatten, d. h. „Staatspolitisch betrachtet wirkt das geltende Frauenwahlrecht antiradikal und konservativ in Kirche und Staat".

Die altbekannte These, erst die Frauen hätten den Wahlsieg Hitlers ermöglicht, muß auf Grund der Wahlstatistik also als falsch zurückgewiesen werden. Interessant ist allerdings, daß sich diese irrige Auffassung bis heute gehalten hat. Die zeitgenössischen Wahlanalytiker waren sich einig, daß das Frauenwahlrecht der NSDAP nicht übermäßig zugute kam. ${ }^{9}$ Von seiten der NSDAP setzte aber schon früh eine Propaganda ein, die den Anschein erweckte, als hätte die Frau wirklich eine überragende Rolle bei den Wahlen gespielt. Ein Beispiel für viele soll genügen: „Daß ich keine Frau genommen habe, hat meinen Einfluß auf den weiblichen Bevölkerungsanteil ständig vermehrt. Ich hätte mir einen Popularitätsverlust bei der deutschen Frau nicht leisten können, denn sie ist doch bei den Wahlen von ausschlaggebender Bedeutung." ${ }^{10}$ Es mag zwar bedauerlich und unverständlich sein, daß es überhaupt Wählerinnen für eine Partei gab, die erklärtermaßen frauenfeindlich war und das aktive und passive Wahlrecht für Frauen ablehnte, das berechtigt aber nicht dazu, der Frau die Verantwortung für die Wahlsiege Hitlers anzulasten.

\section{Die Frauenorganisationen der NSDAP}

\section{a) Die Entwicklung vor 1933}

Von entscheidender Bedeutung für die Bildung von Frauenorganisationen in der Partei vor I 933 waren nicht etwa der Wunsch der weiblichen Mitglieder nach einer Sonderorganisation, sondern die allgemeinen politischen Erfordernisse. Die ersten völkischen Frauenorga-

\footnotetext{
4 Zurkuhlen, S. 545 .

5 So auch Bremme, S. 75 .

6 Zurkuhlen, S. 547.

${ }^{7}$ Hartwig: Wie die Frauen im Deutschen Reich von ihrem Wahlrecht Gebrauch machen. In: Allgemeines Statistisches Archiv. Jena 1928, S. $506 \mathrm{f}$.

8 Zurkuhlen, S. 547.

'Hartwig, S. 506 f. Zurkuhlen, S. 545-547. Max Schneider, S. 73. Bremme kommt in der bercits zitierten Arbeit zu demselben Schluß.

10 Adolf Hitler zit. nach Albert Zoller: Hitler privat. Düsseldorf 1949, S. 106.
} 
nisationen standen lange Zeit außerhalb der Partei. Der „Völkische Frauenorden“ von Elsbeth Zander entsprang nicht der Parteiinitiative, sondern war ein Zusammenschluß völkisch denkender Frauen auf privater Basis. Der Hilfsdienst für die NSDAP wurde erst später - und offensichtlich nicht auf Drängen der Partei - als Programmpunkt aufgenommen. Hitler erkannte 1928 die Arbeit des Ordens an und öffnete damit den Weg zur Zusammenarbeit zwischen Frauenverband und Partei.

Aber obwohl seit dem I.7. 1929 jedes Mitglied des Frauenordens auch der NSDAP beitreten mußte und obwohl der Orden zur Abgrenzung gegen verschiedene völkische Gruppen den Namen „Deutscher Frauenorden“ erhielt, wurde er kein Teil der Partei. Er behielt weiterhin eine eigene Geschäftsführung, eine eigene Reichsleitung, eigene Ortsgruppen und Reichstagungen. ${ }^{1}$ In den Richtlinien heißt es: „Der Orden ist die völkische Frauenbewegung; er treibt nicht selbständig Parteipolitik und steht im Hilfsdienst der Nationalsozialistischen Deutschen Arbeiterpartei unter Führung Adolf Hitlers . . Mitgliedschaft können nur deutschblütige Frauen und Mädchen erwerben, die der N.S.D.A.P. angehören." ${ }^{2}$ Die Bindung an die NSDAP war damit zwar relativ eng, durch die organisatorische Selbständigkeit war der mögliche Spielraum des Ordens aber doch ziemlich groß.

Daneben gab es, wenn man der offiziösen Parteigeschichtsschreibung glauben darf, regionale „Arbeitsgemeinschaften völkisch gesinnter Frauen“ oder örtliche Frauengruppen, die bisweilen sogar im Anschluß an eine NSDAP-Ortsgruppe arbeiteten. ${ }^{3}$ Alle diese Frauengruppen hatten sich aber offensichtlich spontan, ohne das Zutun der Partei gebildet. Erst mit der zunehmenden Verschärfung im Kampf um die Macht und im Hinblick auf die entscheidende Bedeutung der kommenden Wahlkämpfe, stieg das Interesse der Partei an der Frauenarbeit. In der offiziösen Version dieser Vorgänge heißt es: „Die Entwicklung der Frauenarbeit im Dienste der Bewegung, die in den Jahren um 1930 an zahlenmäßigem Umfang erheblich zunahm und aus der Praxis heraus ihre sichtbar sich abzeichnenden vom Wesen der Frau her bestimmten Aufgabengebiete gefunden hatte, drängte aus weltanschaulichen, organisatorischen und arbeitstechnischen Gründen zu einer einheitlichen Führung und Ausrichtung über das Reich hin. "4 Selbst aus diesen floskelhaften Formulierungen läßt sich das Wesentliche noch herauslesen: Nachdem die Partei erkannt hatte, daß die Frauen, in freiwilliger Selbstbeschränkung, keineswegs die Absicht hatten, sich in „Männerangelegenheiten " zu mischen, ging sie daran, die brauchbaren Helferinnen organisatorisch zusammenzufassen und für ihre Zwecke zu schulen. Konsequenz der Überlegungen war die Gründung der NS-Frauenschaft am I.Oktober I93 I. Dieser Organisation, der ersten von der Partei gegründeten Vereinigung von Frauen, gehörten alle weiblichen Parteimitglieder automatisch an. ${ }^{5}$ „Der nationalsozialistischen Frauenschaft (NS-Frauenschaft) ist das Ziel gestellt, im Sinne der Grundsätze der NS-Frauenschaft eine Aktivierung der deutschen Frauenwelt herbeizuführen, die Werbekraft der Frau für die politischen und kulturellen

\footnotetext{
1 BA, NS 22/349.

${ }^{2}$ Richtlinien des Deutschen Frauenordens. S. I.

${ }^{3}$ Nationalsozialistische Frauenarbeit (April r937). S. 93. (BA: NSD 47/1 8).

${ }^{4}$ Ebenda.

${ }^{5}$ Grundsätze der NSF, S. 3.
} 
Ziele der Bewegung einzusetzen und ihre Anlagen und Fähigkeiten einer fürsorgerischen Betätigung innerhalb der Bewegung dienstbar zu machen. ${ }^{6}$

Ưber die fürsorgerische Tärigkeit und ihre Hintergründe ist bereits das Wichtigste gesagt worden. Das gesamte Ausmaß der Hilfsleistungen läßt sich heute nicht mehr genau feststellen. Sicher ist jedoch, daß die Partei mit der geleisteten Arbeit zufrieden war, denn Mißerfolg hätte sicherlich zur Aufhebung oder Umwandlung der Organisation geführt, wie das Beispiel der erfolglosen NSBO zeigt. Die Frauenschaft sollte aber auch propagandistisch tätig werden. Der Hauptakzent bei dieser Arbeit lag auf der „Propaganda im Kleinen“? ? Wenn es auch einzelne Frauenversammlungen gab und einige Rednerinnen ihr Talent vor einem etwas größeren Publikum entfalten konnten, so wurde doch in der Frauenarbeit der Mundzu-Mund-Propaganda das entscheidende Gewicht zugemessen. ${ }^{8}$ Diese Art der Propaganda schien für die Frau angemessen zu sein, die „eigentliche“ politische Arbeit blieb auch weiterhin dem Mann vorbehalten.

Grundsätze und Zielrichtung der Arbeit waren reichseinheitlich in den Richtlinien der NSFrauenschaft festgelegt. ${ }^{9}$ Sie zeigen, daß die Parteileitung die Spitzenfunktionärinnen gut gewählt hatte. Denn obwohl die Grundsätze von Frauen ausgearbeitet wurden, ${ }^{10}$ zeigten sie keinerlei noch so vorsichtige Emanzipationsbestrebungen oder auch nur den Versuch, den Status quo zu verteidigen. Die NS-Frauenschaft zog sich von Anfang an ins Frauenghetto zurück und versuchte sich darin einzurichten. ${ }^{11}$

Neben der grundsätzlichen Orientierung über die Rolle als Frau war es für die propagandistische Tätigkeit notwendig, den Frauen einiges an allgemeinem ideologischen Rüstzeug mitzugeben. Sie mußten in der Lage sein, die Idee des Nationalsozialismus im kleinen Kreis überzeugend darzustellen und Gegenargumente abzuwehren. In einer Art Denkschrift war dazu Grundsätzliches über die Methode festgelegt: „Bei dem Vordringen der weltanschauungsmäßig fundierten Gegner Stennes und Tannenbergbund hat, der Mentalität der Frau entsprechend, die in der Persönlichkeit des Führers ausgeprägte Idee in den Mittelpunkt des Unterrichts zu treten. Die Frau vertraut da unbedingt, wo sie die Idee am reinsten in der Persönlichkeit vertreten sieht. Deshalb wird ein Betrachten des organischen Wachstums der Idee in der Persönlichkeit des Führers zur Festigung der Anhängerschaft führen. Die Darstellung von Weg und Ziel hat möglichst klar und packend zu geschehen. Schwierigkeiten und Blutopfer müssen als selbstverständlich und unbedingt nötig dargestellt werden." ${ }^{12}$ Diese Instruktionen bedeuten nichts anderes, als daß der Frau die Fähigkeit abgesprochen wurde, politische Vorgänge rational zu erarbeiten und zu erkennen. Das verständnismäßige Durchdringen lag allerdings auch nicht im Interesse der Partei - unkritisches, gläubiges Vertrauen und politische Naivität waren ja gerade eine Wurzel des Erfolgs der NSDAP.

\footnotetext{
${ }^{6}$ Ebenda.

7 Grundsätze der NSF, S. 2.

${ }^{8}$ Richtlinien für den „Bund deutscher Mädel“. o. J., S. S, (BA: NS 26/245).

9 Ausführungsbestimmungen, S. 3 .

10 Ebenda.

11 Grundsätze der NSF, S. I f.

12 Hildegard Passow: Propagandistische Erfassung der Frau. S. I, (Herbst 193 I). (BA: NS 26/254).
} 


\section{b) Grundsätzliches zur Frauenfrage nach 1933}

Zum Zeitpunkt der „Machtergreifung“ war die NSF die einzige parteiamtliche Frauenorganisation der NSDAP, ${ }^{13}$ aber keineswegs die einzige existierende Frauenvereinigung. Neben ihr gab es noch zahlreiche andere Frauenverbände, die sich mit ganz unterschiedlichen Zielen und Methoden für die Belange der Frau allgemein oder für eine bestimmte Gruppe von ihnen einsetzten. Die Vielfalt der Organisationen und Zielsetzungen konnte von einem Regime, das die totale Erfassung aller anstrebte, nicht geduldet werden. Nach dem $3 \mathrm{I}$. Januar $\times 933$ mußte also alles daran gesetzt werden, die bereits organisierten Frauen ,zu einer einheitlichen Willensgemeinschaft" ${ }^{\text {"14 }}$ zusammenzuschließen. Darüber hinaus war es aber auch notwendig, die Frauen zu aktivieren und zu erfassen, die noch in ihrer Privatheit verharrten. Mit der Überführung der Frauen in eine nationalsozialistische Organisation war die Eingliederung in das System aber noch nicht beendet. Die Frauen mußten nicht nur erfaßt, sondern auch „ausgerichtet“ werden, d. h. ein umfassender Umerziehungsprozeß war zu leisten. Diese Aufgabe konnte nur von einer Organisation zufriedenstellend gelöst werden, deren Mitglieder fest im NS-Gedankengut verwurzelt waren. ${ }^{15}$ Die NSF erfüllte als einzige Gruppierung diese Voraussetzung - sie wurde zur Führerorganisation erklärt. Parallel dazu begann ab 1933 der Aufbau der entsprechenden Massenorganisation unter Führung der NSF. Bereits aus der Tatsache, daß die organisatorische Arbeit relativ schnell in Angriff genommen wurde und das Gerüst schon Anfang 1934 fertiggestellt war, lassen sich zwei Schliisse ziehen: Die Erfassung möglichst vieler Frauen war offensichtlich ein wirklich dringliches Anliegen, und die Organisatoren trafen bei ihrer Arbeit anscheinend nicht auf nennenswerten aktiven Widerstand bei den betroffenen Frauen.

Der Aufbau der Frauenorganisationen selbst ist gekennzeichnet von einem typischen Phänomen, dem Führergedanken. An der Spitze aller Organisationen, deren Hauptbetätigungsfeld Frauenangelegenheiten waren, stand die Reichsfrauenführerin. Bei ihr liefen alle Fäden zusammen, so daß sie die Arbeit der Frauenorganisationen sinnvoll und planmäßig im Rahmen des Systems einsetzen konnte.

Der hierarchische, am Führergedanken ausgerichtete Aufbau der Frauenarbeit war aber nicht nur als Parallele zum Aufbau anderer nationalsozialistischer Gliederungen gedacht. Gerade für die angestrebte Erziehung der Frau kam ihm noch besondere Bedeutung zu - ein solcher Organisationsaufbau erforderte von den mitarbeitenden Frauen Disziplin und Unterordnung. Das machte die Frauenorganisationen für das Regime wertvoll. Obwohl die Frau in aller Regel von klein auf zu Unterwerfung und Selbstbeherrschung (dem Mann gegenüber) erzogen wurde, so war die reale Unterordnung doch immer noch ein individualistisch-voluntaristischer Akt, der sich sehr stark auf die Person bezog. Straffe Disziplin, wie sie dem Mann z. B. während der Militärzeit anerzogen wurde, war der Frau fremd. ${ }^{16}$ In den Frauenorganisationen sollte nun kein militärischer Drill geübt werden, das empfand man als nicht angemessen. Eine wesentliche Aufgabe der nationalsozialistischen Frauenverbän-

\footnotetext{
${ }_{13}^{13}$ Das galt ab Okt. 1930. Vgl. dazu: Nationalsozialistische Frauenschaft. Berlin 1937, S. 3.

${ }^{14}$ Alice Rilke: 5 Jahre Reichsfrauenführung. In: Die Frau am Werk 4 (1939), S. 50.

${ }^{15}$ Verordnungsblatt der Reichsleitung der NSDAP. o.O. 1933, S. 116, Bekanntgabe von Ley am 19.8.1933.

${ }^{16}$ Gertrud Scholtz-Klink: Einsatz der Frau in der Nation. o. O., o. J., S. 4, (zit.: Scholtz-Klink, Einsatz).
} 
de bestand aber darin, die Frau aus ihrer privaten, individuellen Sphäre herauszulocken und sie bewußt unter das Gesetz der Gemeinschaft zu stellen. Dieser Gemeinschaft und ihren Belangen sollte sie sich fortan unterordnen, die Interessen des Ganzen sollten Maßstab des eigenen Handelns sein. Gelang es, die Frau unter die Gesetze der fiktiven Volksgemeinschaft zu zwingen, so war nicht nur ein wichtiger Schritt ihrer Integration ins System erreicht, sondern es wurde damit bereits die Grundlage für ihre weitere Beherrschung gelegt. Aus der gemeinsamen Unterwerfung unter die Idee des Ganzen wurde propagandistisch die Gleichheit abgeleitet. Daß die emanzipatorische Tendenz des Gleichheitsgrundsatzes der Französischen Revolution durch diesen Gleichheitsbegriff aufgehoben wurde, daß sich hier Gleichheit über solche Inhalte herstellte, die die grundsätzliche Gleichheit des Menschen ausdrücklich zurücknahmen, spielte dabei offenbar keine Rolle. Die Quadratur des Kreises schien gelungen - die Gleichheit aller, die seit Jahrzehnten als Ideal des Menschen galt und die seit langem von verschiedenen politischen Parteien gefordert und versprochen worden war, existierte nun plötzlich. Es gab - propagandistisch - nicht mehr die Akademikerin, die Arbeiterin oder die Bäuerin, es existierten nur noch Frauen, die sich dem Gesetz der Gemeinschaft unterwarfen und für diese Gemeinschaft tätig waren.

Auffallend in der Geschichte der NS-Frauenverbände ist, daß die offiziöse Geschichtsschreibung zwar recht ausführlich von der Frauenarbeit der „Kampfzeit“ und der Jahre ab I 934 berichtete, die Ereignisse von 1933 hingegen nicht berücksichtigte. Zwei Gründe dürften für die Streichung des Jahres 1933 aus den Annalen der nationalsozialistischen Frauenorganisation ausschlaggebend gewesen sein. Zunächst kam es zu einer Personaldebatte, die erst im Februar 1934 mit der Ernennung der badischen Gauführerin ScholtzKlink zur Führerin der Frauenverbände abgeschlossen wurde. ${ }^{17}$ Ein weiteres Problem bestand darin, daß auch die Frauenorganisationen Zeit brauchten, um ihre endgültige Form zu erhalten. Die Organisation war dabei keineswegs aus einem Guß, es war vielmehr klar erkennbar, daß der Aufbau der Massenorganisation nicht nach einem streng durchdachten, bereitliegenden Plan erfolgte. Zudem bedurfte es massiver Zwangsmittel, um die bis 1933 bestehenden Frauenvereinigungen aufzulösen und in die NS-Massenorganisation zu überführen. All diese Schwierigkeiten offen zuzugeben wäre nach dem Selbstverständnis der Nationalsozialisten gleichbedeutend gewesen mit dem Eingeständnis der Schwäche und Unzulänglichkeit. Da die Entwicklung der Frauenorganisationen nicht im Mittelpunkt des öffentlichen Interesses standen, war es möglich, die Probleme der Anfangsphase einfach zu ignorieren.

\section{c) Nationalsozialistische Frauenschaft und Deutsches Frauenwerk}

Wie in anderen Bereichen auch, ging man vor der Neuorganisation der Frauenarbeit zunächst daran, die nicht-nationalsozialistischen Frauenverbände zu zerstören oder „gleichzuschalten“. Gleichschaltung bedeutete, daß der Verein zwar formal weiterbestand, in seiner Funktionsfähigkeit aber stark beschränkt wurde. Er mußte sich bedingungslos dem Führer der NSDAP unterordnen und die Forderungen, die der NS-Staat an ihn stellte, ak-

17 Rilke, S. so. A.O. über die Beurlaubung von E. Zander und die Ernennung von L. Gottschewski in Verordnungsblatt der Reichsleitung der NSDAP I 933, S.97, (Ley am 26.4. I 933). 
zeptieren. Um die Durchsetzung dieser Prinzipien zu garantieren, wurden nationalsozialistische Frauen in die Vorstände der Organisationen "gewählt". ${ }^{18}$ Wenn es sich nicht gerade um parteipolitisch gebundene Frauenverbände handelte, deren Auflösung mit dem „Ende der Parteien " einherging, ${ }^{19}$ so wurde offenbar der Versuch gemacht, möglichst viele Frauenvereine „gleichzuschalten“. Dieses Vorgehen hatte verschiedene Vorteile: Die Gelder der Verbandskassen konnten mit „überführt“ werden, das hohe Ansehen, das einige der alten Organisationen genossen, war möglicherweise - bei geschickter Regie - auf den Gesamtverband übertragbar und die Frauenorganisationen stellten einen Stamm praktisch orientierter, erfahrener Frauen, die für die Aufbauarbeit unentbehrlich waren. Wie wichtig gerade die letzte Funktion der alten Frauenverbände war, zeigt sich an den wiederholten Ermahnungen, Erfahrungen und Kenntnisse in die Fraueraarbeit mit einzubeziehen, die von Frauen gewonnen wurden, die noch keine Nationalsozialistinnen waren. ${ }^{20}$

Die „gleichgeschalteten“ Vereine wurden dem neu geschaffenen Deutschen Frauenwerk (DFW) als „angeschlossene Verbände“ unterstellt. Aber nicht nur Gesamtverbände gehörten zum DFW. „Jede deutsche Frau arischer Abstammung (bei verheirateten Frauen gilt das gleiche für den Ehemann) kann Einzelmitglied“21 werden, um „ihre Arbeit und ihre Fähigkeiten ... in den Dienst der Gemeinschaft unseres Volkes zu stellen." ${ }^{22}$ An anderer Stelle hieß es: „Die Mitglieder des Deutschen Frauenwerks sind zwar nicht Mitglieder der NSDAP, bzw. der N.S. Frauenschaft, aber sie bekunden mit ihrer Mitgliedschaft beim Deutschen Frauenwerk ihre Verbundenheit mit der nationalsozialistischen Idee, indem sie sich der nationalsozialistischen Führung unterordnen." ${ }^{23}$

Der „Dienst“, den die Frau im DFW zu leisten hatte, war entsprechend den Zielen der neuen Organisation ein doppelter: unter Leitung der NSF wurden die Mitglieder ideologisch geschult und für praktische Arbeiten eingesetzt. Die Unternehmungen des DFW waren zwar hauptsächlich wohlfahrtspflegerisch-fürsorglicher Art, die Lösung dieser Aufgaben entsprang aber keineswegs einem sozialen Verantwortungsbewußtsein des Regimes. Die Arbeit war vielmehr von eminent politischer Bedeutung. Sie diente zunächst eindeutig dazu, das Image des neuen Staates zu verbessern, gleichzeitig war sie aber auch notwendig, um die stark eingeschränkte karitative Arbeit der Kirchen und der aufgelösten Frauenverbände zu ersetzen. Die angeschlossenen Verbände und auch die Einzelmitglieder leisteten oft dieselbe Arbeit wie vorher, der Erfolg wurde aber auf das Konto der NS-Organisationen gebucht. Mit Hilfe dieses Etikettenschwindels versuchte das Regime, die Bevölkerung von seiner sozialen Einstellung zu überzeugen und sie auf diese Art für sich zu gewinnen. Gleichzeitig

\footnotetext{
${ }^{18}$ Leonore Kühn: Auflösung. In: Die Deutsche Kämpferin I (1933/34), S. 4 I, (zit.: Kühn, Auflösung). Die hier beschriebenen Bedingungen können wohl als exemplarisch angesehen werden.

19 Nationalsozialistische Frauenschaft, S. 16.

${ }^{20}$ Krummacher: Organisation, Aufgaben und Pflichten der Nationalsozialistischen Frauenschaft. In: Presseamt der Reichsleitung der NS Frauenschaft (Hrsg.): Nationalsozialistische Frauenkorrespondenz I 933 , Nr. 40, I. I I. I 933 , (zit.: Krummacher, Organisation). (s. dazu Anhang II).

21 Nationalsozialistische Frauenschaft, S. 19.

22 Ebenda, S. 20.

${ }^{23}$ Krummacher: Deutsches Frauenwerk. In: Nachrichtendienst: Deutsches Frauenwerk Nr. 6, I s. x. 1934, (BA: NSD 39), (zit.: Krummacher, Frauenwerk).
} 
fand auf diesem Weg eine gewisse Indoktrination statt, die wesentlich gefährlicher (weil schwer durchschaubar) war als jede marktschreierische Propaganda.

Die praktische Arbeit, die im engen Zusammenhang mit den alltäglichen Aufgaben der Hausfrau stand, war ebenfalls ein bewußt eingesetztes Mittel, um gerade die nicht berufstätige Frau zu erfassen. Folgende Ưberlegung stand dahinter: „Es ist zu allen Zeiten ein Problem gewesen, Frauen zu organisieren, und wird es vielleicht immer bleiben; der Grund dürfte darin zu suchen sein, daß die Frau weniger durch äußere Disziplin und Kommandos zu erfassen ist als der Mann, und daß sie alle Dinge in eine ganz nahe Bindung zu ihrem eigenen täglichen Lebenskreis bringen muß, wenn sie sich dafür einsetzen soll, . . es wird deshalb die Frage des Einsatzes der Frau einer Nation . . . dort im selben Maß an Schwierigkeiten verlieren, wo es am besten gelingt, die großen, tragenden Ideen einer Volksführung dem alltäglichen Lebensbereich der Frau so verständlich zu machen, daß sie ihre Verwirklichung als eigene Aufgabe ansieht, und sie dann auch mit der ihr eigenen Gläubigkeit und Zähigkeit verfolgt. " ${ }^{24} \mathrm{Ob}$ diese Rechnung aufging, wird zu prüfen sein.

Um dem Anspruch nach zielklarer und wohlgegliederter Arbeit ${ }^{25}$ gerecht zu werden, setzte sich das Frauenwerk aus verschiedenen Hauptabteilungen zusammen. „Den deutschen Frauen als den ,Müttern der Nation' galt die erste Sorge“ ${ }^{26}$ Daher wurde zuerst der Reichsmütterdienst eingerichtet, der sich mit der Ausbildung der zukünftigen Mutter befaßte: „Die Herausbildung von körperlich und seelisch tüchtigen Müttern, die überzeugt sind von den hohen Pflichten der Mutterschaft, die erfahren sind in der Pflege und Erziehung ihrer Kinder und die ihren hauswirtschaftlichen Aufgaben gewachsen sind . . ist seine wichtigste Aufgabe." ${ }^{27}$ In Lehrgängen, denen zum ersten Ma! seit den Anfängen der Frauenbewegung ein reichseinheitlicher Rahmenlehrplan zugrunde lag, ${ }^{28}$ wurde die Frau in den wichtigsten Fertigkeiten unterrichtet. Ähnliche Veranstaltungen führte auch die 2. Hauptabteilung, die Abteilung Volkswirtschaft/Hauswirtschaft durch. Hier wurden offenbar nicht nur praktische Kenntnisse der Haushaltsführung u.ä. vermittelt, sondern auch ein neues Selbstbewußtsein für Hausfrauen. Der Leitgedanke dieser Abteilung war „Wir dienen dem Leben unseres Volkes. Wir betrachten unsere hausfrauliche Arbeit als Mittel zur Erreichung und Erhaltung der Gesundheit unseres Volkes an Leib und Seele aus den Kraftquellen unserer eigenen Volkswirtschaft." 29 Besonders der letzte Aspekt gewann im Zusammenhang mit dem Vierjahresplan immer mehr an Bedeutung. Die Erziehung zu rationeller Rohstoffverwertung und die Verbrauchslenkung rückten bald in den Mittelpunkt der Arbeit.

Zur ideologischen Unterstützung und einheitlichen Ausrichtung der Arbeit gab die Abteilung Presse/Propaganda sowohl eigene Zeitschriften heraus, wie die Nationalsozialistische Frauenwarte, als auch Beilagen zu verschiedenen Gauzeitungen. Die eigentliche propagandistische Arbeit wurde aber in den Lehrgängen selbst geleistet.

\footnotetext{
${ }^{24}$ Gertrud Scholtz-Klink: Aus der Rede vor der Reichsfrauenschaft (Reichsparteitag). In: Die Frau 45 (1937/38), S. 45, (zit.: Scholtz-Klink, Reichsparteitagsrede I937).

${ }^{25}$ Krummacher, Frauenwerk, S. I .

26 Nationalsozialistische Frauenschaft, S. 20.

27 Ebenda, S. 2 I.

${ }^{28}$ Ebenda, S. 22.

29 Ebenda, S. 24.
} 
Bei der Beantwortung der Frage, wie erfolgreich im Sinne des Systems die Arbeit der Abteilungen wirklich war, lassen sich nur Vermutungen anstellen. Die Teilnehmerzahlen der Kurse sind zwar relativ hoch, sie sind aber nicht unbedingt identisch mit der Zahl der tatsächlich erreichten Frauen. Da die Lehrgänge jeweils ganz spezielle Themen hatten, war es eben auch sinnvoll, wenn eine Frau verschiedene Kurse mit unterschiedlichen Schwerpunkten besuchte. Die Teilnahme selbst sagt gerade bei den praktisch orientierten Veranstaltungen wenig über das Verhältnis der Frauen zum Regime oder zu NSF/DFW. Auch der Besuch von Kultur- und Sportveranstaltungen, die von der Abteilung Kultur, Erziehung, Schulung ausgerichtet wurden, besitzt in diesem Sinne keine Aussagekraft. Festzuhalten bleibt aber, daß ganz offensichtlich eine Mobilisierung und Aktivierung der Frauen gelungen war, was dem Ziel der totalen Erfassung nur förderlich sein konnte.

Die Mobilisierung, die sich bei der Teilnahme von Veranstaltungen und Kursen zeigte, läßt sich auch bei der Betrachtung der Mitgliederzahlen feststellen. Die NSF hatte, nach eigenen Angaben, Ende 1938 im Altreich 2294677 Mitglieder, das DFW I 783335 selbständige und rund 4 Millionen korporative Mitglieder, ${ }^{30} \mathrm{~d}$. h. etwa I 3,2\% aller deutschen Frauen über 20 Jahre waren in einer NS-Frauenorganisation erfaßt. ${ }^{31}$

Auf den ersten Blick verblüfft besonders das Verhältnis NSF - DFW. Die NSF, die als Eliteorganisation aufgebaut worden war, hatte ganz eindeutig mehr Einzelmitglieder als das DFW, die Massenorganisation. Begünstigt wurde der Zustrom zur NSF in der Anfangsphase wohl vor allem dadurch, daß der Beitritt ab I 933 nicht mehr an die Parteimitgliedschaft in der NSDAP gekoppelt war. Die psychische Hemmschwelle, die gegenüber einem Parteieintritt bei den Frauen offensichtlich sehr hoch war, wurde so umgangen. Das ist ein Zeichen dafür, daß dringend möglichst viele Frauen gebraucht wurden, die sich aktiv an der Aufbauarbeit beteiligten, es wird aber auch deutlich, daß die NSDAP selbst nach 1933 ihren Charakter als „Männerpartei“ nicht verlor und nur ein geringes Interesse an der Neuaufnahme von Frauen hatte. ${ }^{32} 1936$ wurde der Zufluß zur NSF durch eine Anordnung von Heß gestoppt: „Die NS.-Frauenschaft hat inzwischen einen Mitgliederstand erreicht, der zur Lösung der ihr als Führerinnenorganisation der deutschen Frauen gestellten Aufgaben vollkommen genügt. Ich bestimme daher, daß die weitere Aufnahme in die NS-Frauenschaft am I. Februar 1936 gesperrt wird." ${ }^{33}$ Danach war die Mitgliederbewegung zwar leicht rückläufig, der Mitgliederstand lag aber weiterhin eindeutig über dem des DFW. Erklärbar ist diese Entwicklung durch die Aufnahmemodalitäten nach dem I. 2. 1936. Führerinnen des BDM, des Frauenarbeitsdienstes und des DAF-Frauenamts waren von der Anordnung nicht betroffen, ${ }^{34}$ sie konnten auf Antrag auch weiter in die NSF aufgenommen werden, d. h. auf bewährte Führerinnen wollte man nicht verzichten.

${ }^{30}$ Reichsfrauenführung (Hrsg.): NS Frauenschaft und Deutsches Frauenwerk. Statistischer Bericht. Jahresbericht 1938. S. I. (IfZ: $\mathrm{Db}$ 40.10). Von den unterschiedlichen Zahlen wurden die jeweils höchsten genommen.

${ }^{31}$ Ebenda, S. 19.

${ }^{32}$ Ebenda, S. 17. Danach waren am I. I. 1939 6,39\% der DFW/NSF Mitglieder im Altreich gleiclizeitig Parteimitglieder.

${ }_{33}$ Verordnungsblatt der Reichsleitung der NSDAP 1 936, S. 377.

${ }^{34}$ Ebenda. 
Auf der Grundlage der Mitgliederzahlen zu den Frauenorganisationen Rückschlüsse über die Stellung der mitarbeitenden Frauen zum Regime zu ziehen ist schwierig. Denn während man für die Kampfzeit der Partei noch davon ausgehen kann, daß die in der NSF organisierten Frauen der „Bewegung“ positiv gegenüberstanden und die Arbeit der Partei bewußt unterstützen wollten, so ändert sich das Bild nach 1933 schlagartig. Neben gewissen Erwägungen der Zweckmäßigkeit (sprich Opportunismus) und der Zwangsauflösung vieler Frauenverbände darf das karitative Motiv nicht übersehen werden. In Unkenntnis des verbrecherischen Charakters des Regimes, geblendet von dem sozialen Aktivismus der Organisationen glaubten sicherlich viele Frauen, hier ein passendes Betätigungsfeld gefunden zu haben. Aus dem hohen Organisationsgrad der Frauen im NS-Staat läßt sich so zwar nicht unmittelbar Zustimmung zum Regime ableiten, objektiv hatte die Aktivierung so vieler Frauen und ihre praktische Tätigkeit aber eine gewisse Integrationsfunktion und wirkte damit systemstabilisierend. ${ }^{35}$

Die NS-Frauenorganisationen waren aber nicht nur Mittel zur Erfassung, theoretisch boten sie auch die Chance zur Emanzipation der Frau. Die große Mitgliederzahl und die Wichtigkeit der Arbeit für das System waren eine gute Ausgangsposition, um frauenspezifische Forderungen zu stellen. Zudem war nun die wesentliche Voraussetzung erfüllt: Ein Teil der Frauen war dem engen Lebensbereich der Hausfrau und Mutter entkommen und konnte zusammen mit anderen Frauen darangehen, die eigene Situation zu erkennen und zu verändern. Bedauerlicherweise blieb es bei der theoretischen Möglichkeit. Bis auf einen kleinen Kreis von Kritikerinnen, die relativ schnell mundtot gemacht wurden, gab es keine sichtbaren Versuche, sich aus der traditionellen Frauenrolle zu lösen. ${ }^{36}$

Die Entfaltungsmöglichkeiten der Frauen waren durch ständige Überwachung und Kontrolle allerdings auch stark eingeschränkt. Das System der permanenten gegenseitigen Kontrolle, das bewußt als Herrschaftsmittel eingesetzt wurde, verhinderte weitgehend die notwendige Solidarität unter ihnen. Daß die Überwachung als Fürsorge getarnt wurde, änderte nichts an der Tatsache, ${ }^{37}$ sondern zeigt nur nochmals das zynische Verhältnis des Regimes zum Menschen. Eine zusätzliche Schwierigkeit lag im Aufbau der Organisation selbst. Die straffe hierarchische Führung, die nur Befehle nach unten und Verantwortung nach oben kannte, schuf sicherlich kein Klima, das der Entwicklung persönlicher Freiheit und der Entwicklung kritischen Denkens zuträglich gewesen wäre. Zudem stand auch die Führung unter ständiger Aufsicht, die Angelegenheiten der Frauen lagen keineswegs in ihrer eigenen Verantwortung - „typisch frauliche Aufgaben“ wurden ihnen zwar überlassen, konkret politische Aktivitäten aber waren Männern vorbehalten. Männer hatten auch über das Verhalten der Führerinnen zu entscheiden, die Frauenschaftsleiterinnen waren auf jeder Ebene den entsprechenden Leitern disziplinär unterstellt. ${ }^{38}$ Selbst die Reichsfrauenführerin hatte

\footnotetext{
${ }^{35}$ Die damit verbundene politische Naivität ist nicht frauenspezifisch und muß daher auch nicht thematisiert werden.

${ }^{36}$ Möglicherweise war dies im Widerstand anders.

${ }_{37} \mathrm{Zu}$ dieser Ưberwachung vgl. z. B. die Aufgaben der Blockleiterinnen: Aufgaben der Block- und Zellenfrauenleiterin. (BA: NSD 47/I 8. Dezember I 937), S. 332-334.

${ }^{38}$ Reichsorganisationsleiter der NSDAP (Hrsg.): Organisationsbuch der NSDAP. München I940', S. 271.
} 
noch einen Vorgesetzten, ${ }^{39}$ der die politische Linie des Verbandes nach Anweisung der Reichsleitung der NSDAP bestimmte. ${ }^{40}$ Das wichtige Amt der Finanzverwaltung wurde ebenfalls von einem männlichen Parteigenossen verwaltet. ${ }^{41}$

Offenbar gab es wegen der Unterordnung der Frauenorganisation unter eine männliche Leitung einige Probleme, denn die NS Frauenwarte, die von der Reichsfrauenführung herausgegeben wurde, sah sich veranlaßt, diesen Tatbestand zu erklären. Danach bedeutete die Unterstellung der Frauenbewegung unter männliche Herrschaft nichts anderes als die Anerkennung der Einheitlichkeit der Bewegung. Frauenfragen durften keine Domäne der Frau sein, sondern auch der Mann war davon betroffen. ${ }^{42}$ Warum dies nicht auch umgekehrt der Fall sein konnte, wurde nicht weiter erläutert. Ob es sich bei dieser Aussage nun um die wirkliche Meinung der Reichsfrauenführung handelte oder nicht, sei dahingestellt, jedenfalls kam dadurch zum Ausdruck, daß die Führung selbst bereit war, sich mit einer untergeordneten Stellung und geringem Einfluß zufriedenzugeben. Eine Unterstützung eventuell vorhandener Emanzipationsbestrebungen war von ihr nicht zu erwarten.

\section{d) Frau und Kirche}

Zu klären bleibt, wieweit die Bemühungen des NS-Regimes, das religiöse Empfinden der Bevölkerung umzuorientieren, erfolgreich waren. Eine gewisse Beharrungstendenz zeigt sich bereits bei den Wahlen zum evangelischen Kirchenparlament Thüringens im Januar 1933. Die NSDAP konnte bei der Wahl des Landeskirchentages, der durch Urwahlen bestellt wurde, nur 67000 Stimmen auf sich vereinigen, während sich bei den politischen Wahlen im Vorjahr immerhin 400000 Wähler für sie ausgesprochen hatten. ${ }^{43}$ Offensichtlich unterschieden die Wähler also sehr genau zwischen politischen und kirchlichen Belangen.

Ein Versuch, in das kirchliche Leben einzugreifen, war die Uminterpretation des Erntedankfestes. Besonders auf dem Land aber hielt die Bevölkerung an der christlichen Tradition fest; fanden gleichzeitig Erntedankveranstaltungen von Partei und Kirche statt, entschieden sich die meisten für den Kirchgang. ${ }^{44}$ Auch bei den Lebensfeiern mußte das Regime Mißerfolge hinnehmen. Vor allem die gescheiterte Ersetzung von Erstkommunion bzw. Konfirmation durch HJ-Verpflichtungsfeiern ${ }^{45}$ wog schwer, denn hier zeigte sich mit aller Deutlichkeit, daß die Aufnahme der Kinder in die Kirchengemeinde den meisten Eltern wichtiger war als die Aufnahme in die politische Gemeinde.

Besonders das Verhältnis der Frauen zur Kirche machte dem Regime offenbar zu schaffen. Aus den Monatsberichten der NS-Frauenschaft geht hervor, daß das religiöse Potential bei

\footnotetext{
${ }^{39}$ Nach Robert Ley (Hrsg.): Nationalsozialistisches Jahrbuch 1939. München o. J., S. 238 war Hilgenfeld der zuständige Amtsleiter.

${ }^{40}$ Die Frau 40(1933), Heft I, S. 506.

${ }^{41}$ Zum Organisationsplan der NS-Frauenschaft/Deutsches Frauenwerk. In: Jahrbuch der Reichsfrauenführung 1938 , S. I 4 .

${ }^{42}$ Lydia Gottschewski: weibliches Führertum. In: FW 2 (1933/34), S. 179, (zit.: Gottschewski, weibliches Führertum).

${ }^{43}$ Dr. A: Lehrreiche Wahl in Thüringen, In: Osnabrücker Volkszeitung 27. I. 1933.

${ }^{44}$ Klaus Vondung: Magie und Manipulation. Göttingen 1971, S. 106.

${ }^{45}$ Ebenda, S. 105.
} 
den Frauen noch recht stark war und bewußt von der Kirche angesprochen wurde. ${ }^{46}$ „Die Pfarrer haben sehr wohl erkannt, daß sie nur durch Gewinnung der Frauen ihren Einfluss in Familie und Volk aufrecht erhalten können “, ${ }^{47}$ klagte die NSF Schlesiens, und der Gau Hessen-Nassau stellte fest: „Von diesen Seiten wird mit allen Mitteln versucht, die Frau für sich zu gewinnen. Man hofft, über die Frauen die Kinder und teilweise die Männer wieder unter kirchlichen Einfluß zu bekommen." ${ }^{48}$ Dies war der entscheidende Punkt; vor diesem Hintergrund ist zu verstehen, daß sich die zuständigen Frauenorganisationen so stark mit dem Problem auseinandersetzten und so großes Interesse daran hatten, die Frau aus ihren kirchlichen Bindungen zu lösen.

Der Erfolg dieser Bemühungen war recht wechselhaft. Gelang es zunächst, auch Teile der konfessionell gebundenen Frauen in der NSF zu organisieren, so gab es bereits 1936 wieder Austritte in größerem Maße, was die Frauenschaft auf die ungelöste Kirchenfrage zurückführte. ${ }^{49}$ Andere Teile der weiblichen Bevölkerung wurden durch die propagandistischen Appelle gar nicht erreicht. Die Gründe für das Dilemma der Frauenschaft waren unterschiedlich. Das Frauenwerk der Deutschen Evangelischen Kirche und die Evangelische Reichsfrauenhilfe hatten sich aus der Reichskirche gelöst und sich auf die Seite der Bekennenden Kirche gestellt. ${ }^{50}$ Ihre Mitglieder dürften daher wohl keine geeigneten Adressaten für die NSF gewesen sein. Im katholischen Bereich erwiesen sich vor allem die Angriffe auf Priester als ungünstig für die Frauenagitation. So bekannte der Gau Westfalen-Nord: „Die Frauen verschließen sich in immer stärkeren Maßen der Bewegung." Der Bericht führte diese Entwicklung hauptsächlich auf die Sittlichkeitsprozesse gegen Priester zurück, die nicht zu Unrecht als Angriff auf die Kirche gewertet wurden. ${ }^{51}$ Vor allem aber rief die antikirchliche Schulpolitik der Regierung Widerstand hervor. ${ }^{52}$

Die vorsichtige Zurückhaltung der nationalsozialistischen Frauenzeitschriften in religiösen Fragen $^{53}$ und die Anweisung der Reichsfrauenführerin, Auseinandersetzungen in diesem Bereich grundsätzlich zu vermeiden, ${ }^{54}$ machten sich offensichtlich nicht bezahlt. Die Frauen bezogen ihre Bewertungsmaßstäbe für den religionspolitischen Kurs des Regimes offenbar aus anderen Quellen.

Eine der wichtigsten Instanzen dürfte, gerade auf dem Land, der Pfarrer gewesen sein. Die Verurteilung der antikirchlichen Regierungspolitik von der Kanzel aus ließ viele Frauen wohl vor einer näheren Auseinandersetzung zurückschrecken. Die geistige Unselbständigkeit und die daraus resultierende geistige Unflexibilität, die der Frau durch die Kirche jahrhundertelang systematisch anerzogen worden war, erwies sich nun für die Kirche selbst als

\footnotetext{
${ }^{46}$ Monatsberichte des Hauptamtes NS Frauenschaft für die Monate Juli/August I 935, S. 2-6, (BA: NS 22/860).

47 Monatsbericht für Juni 1937, S. I 9.

48 Monatsbericht für November 1937, S. 1 3.

49 Monatsbericht für Juli/August I936, S. I.

50 Die Deutsche Kämpferin 3 (1935/36), S. i I 3.

51 Monatsbericht für Juli/September 1937, S. 24.

52 John S.Conway: Die nationalsozialistische Kirchenpolitik 1933-1945. München 1969, S. 203. Der Höhepunkt war die Ersetzung des Kruzifixes durch Hitlerbilder.

53 Vgl. dazu z. B. FW.

${ }^{54}$ Monatsberichte für September/Oktober I 936, S. 4 .
} 
ausgesprochen positiv. Die Frauen hörten weiterhin auf die Belehrungen der Pfarrer und ließen sich z. T. eben nicht organisieren. Um praktisch-politisch wirksam zu werden, hätte die Verweigerung aber weiter gehen müssen. Da sie nur partiell war, blieb sie insgesamt politisch wirkungslos.

Aber auch wenn sich die Frauen in einem kleinen Bereich dem Regime verweigerten, so heißt das noch nicht, daß die Bemühungen zur Veränderung des religiösen Bewußtseins als totaler Mißerfolg zu werten sind. Denn bei genauerer Betrachtung läßt sich feststellen, daß der Erfolg dort besonders gering war, wo es sich um Eingriffe und Übergriffe ins kirchliche Leben und seine Ausdrucksformen handelte. Dies läßt sich aber mit normalen menschlichen Beharrungstendenzen, sprich: Festhalten an Traditionen, erklären; eine Aussage über die emotionale Haltung ist nicht unbedingt darin eingeschlossen. Da Nachrichten über unterbewußte psychische Strukturen naturgemäß fehlen, lassen sich nur Vermutungen anstellen. Geht man davon aus, daß selbst Regimegegner sich von den öffentlichen Kundgebungen, Appellen etc. emotional angesprochen fühlten und die ungeheure Faszination verspürten, die von diesen Massenveranstaltungen ausging, ${ }^{55}$ so kann man wohl unterstellen, daß Menschen, denen die kritische Distanz zum Regime fehlte, diesen Appellen an die Emotion erlagen, d.h. sie wurden in einen Zustand versetzt, in dem die rationale Selbstkontrolle weitgehend ausfiel. Diese Bewußtseinstrübung eignete sich natürlich hervorragend zur Indoktrination. Hinzu kam, daß bestimmte Parolen und Ideen ja Tag für Tag auf die Bevölkerung niederprasselten, so daß sicherlich einiges hängenblieb.

Betrachtet man weiterhin den ungeheuren Einsatz, auch der Zivilbevölkerung, im Krieg und die Entbehrungen, die die Bevölkerung offensichtlich bereit war zu tragen, was nicht allein durch brutale Unterdrückungsmaßnahmen erklärt werden kann, so liegt die Vermutung nahe, daß der Propaganda doch gewisse Teilerfolge bei den Betroffenen gelungen sind.

\section{Kritische Stimmen der Bewegung}

Trotz aller Unterdrückung, trotz Zensur und ständiger Kontrolle und trotz aller zur Schau gestellten Einheitlichkeit nach außen erhob sich gegen das Frauenbild und die praktischen Konsequenzen dieser Ideologie recht lautstarke Kritik von Zeitgenossinnen, die der Idee des Nationalsozialismus durchaus positiv gegenüberstanden. Besonders der Kreis um die „Deutsche Kämpferin“ protestierte sehr vehement gegen Bestrebungen, „die Frau wiederum zum schlichten Objekt männlicher ,Benutzung“ herabzudrücken“.' Die Mitarbeiterinnen dieses Blattes wandten sich mit ihrem Protest direkt an Adolf Hitler. Die Kritik stieß offenbar auf starkes Interesse, denn bereits 1934 erschienen die publizierten Protestschreiben in der dritten Auflage. ${ }^{2}$

Von offizieller Seite erfolgte zunächst keine Reaktion, doch als die kritischen Stimmen nicht freiwillig verstummten, wurde die „Deutsche Kämpferin“ im Juni 1937 kurzerhand

\footnotetext{
${ }_{55}$ Victor Klemperer: LTI. Frankfurt a. M. 1975 (1946), S. 290.

1 Leonore Kühn: Geistige Führung im Frauentum. In: Die Frau 4I (I 933/34), S. 2 I I , (zit.: Kühn, Führung).

${ }^{2}$ Theodore Eichhoff: Nationalsozialistische Stimmen zur Frauenfrage. In: Soziale Praxis NF 43 (1934), Sp. 1244.
} 
verboten. ${ }^{3}$ Die erstaunliche Langmut des Regimes ist wohl nur mit dem geringen Einfluß der Zeitschrift zu erklären. Die Kritikerinnen wehrten sich gegen das Zurückdrängen der Frau aus der Politik und versuchten, in ihrer Argumentation die Nationalsozialisten mit deren eigenen Waffen zu schlagen: Geht man davon aus, daß die Frau anders geartet ist als der Mann, so kann sie von ihm nicht politisch vertreten werden, und muß selbst an den Entscheidungsprozessen teilnehmen, ist sie aber gleichartig, so gibt es auch keinen Grund, sie nicht an den politischen Entscheidungen zu beteiligen. ${ }^{4}$

Aućh die Zurückführung der Frau an Heim und Herd wurde scharf verurteilt: „Einerseits hebt man die Frau auf den Thron; dort darf sie madonnenhaft lächeln, aber nicht hinuntersteigen in diese irdische Welt, um verantwortungsvoll zu handeln ", ${ }^{5}$ andererseits dürfe sie aber die schwere Hausarbeit verrichten oder sich ihren Lebensunterhalt in „arteigenen “ Berufen verdienen, die sich dadurch auszeichnen, daß sie die Frauen begrenzen und meist wesentlich unangenehmer sind als die, die von Männern bevorzugt werden. ${ }^{6}$ Gerade den Frauen, die höhere, verantwortungsvolle Positionen anstrebten, würden Steine in den Weg gelegt, während das bei Arbeiten, wo Frauenarbeit billige Arbeitskraft bedeute, nicht der Fall sei. ${ }^{7}$ Mit dem Ausschluß aus anderen einflußreichen und geistig fordernden Berufen, würde die Frau als unfähig und minderwertig erklärt, und „darüber täuscht auch keine Verherrlichung hinweg ". ${ }^{8}$ Selbst die Verherrlichung der Mutter diene nicht der Würdigung der Frau, sondern die hoch gepriesene Mutter diene letztlich nur als Mittel zum Sohn, die Tochter als Mittel zu weiteren Söhnen, „beide nur als Mittel zum Volk der Söhne“, ${ }^{9}$ von denen dann die besten zu Führern der Volksgemeinschaft bestimmt würden. ${ }^{10}$

\section{Die Frau im Kulturleben}

\section{Frau und Kunst}

\section{a) Die Frau als Künstlerin}

„Es ist der Frau im allgemeinen nicht wie dem Manne gegeben, das, was sie innerlich bewegt, künstlerisch $z u$ gestalten, und damit für andere Menschen mit erlebbar zu machen." Ihr spezifischer Kulturbeitrag besteht vielmehr „im Dienst am Menschen in den

\footnotetext{
${ }_{3}$ Die Deutsche Kämpferin, Juniheft I937, (laut Umschlagheft handelt es sich zwar um ein Heft des Jahres 1936, das muß aber ein Druckfehler sein).

${ }^{4}$ Irmgard Reichenau: Die begabte Frau. In: Dies. (Hrsg.): Deutsche Frauen an Adolf Hitler. Leipzig o. J. (1933), S. 23.

5 Ebenda, S. 26.

6 Ebenda, S. 20.

7 Eva Eberstein: Um unsere Einigkeit! Leipzig I934, S. 66.

8 Reichenau, S. 2. $x$.

9 Leonore Kühn: Natürlicher Aristokratismus. In: I. Reichenau, S. 37, (zit.: Kühn, Aristokratismus).

10 Kühn, Aristokratismus, S. 29.

1 Nationalsozialistische Parteikorrespondenz: Sonderdienst. Die deutsche Frau, 1 8.6. 1934. - Im folgenden handelt es sich um grundsätzliche Uberlegungen. Im Mittelpunkt steht nicht das künstlerische Werk einzelner, sondern es soll versucht werden, die Verfügbarkeit der Kunst für das System und die Begren-
} 
mannigfachen Formen und in der Gemeinschaftsbildung“.2. Erst "die nicht durch den Dienst an der Erhaltung der Art und durch den Dienst am Menschen aufgezehrte Kraft der Frau vermag sich ... wie die männliche in objektiven Kulturgütern, in Werken darzustellen, " ${ }^{3}$ wobei die natürliche mütterliche Kraft ihr Schaffen aber doch stets bestimmen müsse. ${ }^{4}$ „Die schöpferische deutsche Frau muß Sorge tragen, daß dieser ewige Ruf nach wahrer Mütterlichkeit seine hohe Bedeutung behält, daß die Frau immer im höchsten Sinn Frau bleibt. Ist's doch gerade ihr, der Frau gegeben, das Wesen alles Bestehenden tief zu erfüblen, und auch das Werdende, Zukünftige ahnungsvoll vorauszuempfinden. Nur die Künstlerin, die wahrhaft Frau geblieben, vermag in ihrem Kontakt zur Umwelt ihres Amtes als Erzieherin im schönsten Sinn zu walten."

Trotz des Verdikts, die Frau „,im allgemeinen“ sei nicht zu künstlerischer 'Tätigkeit berufen, gab es natürlich auch im Dritten Reich Künstlerinnen, und auch die wenigen großen Namen der Vergangenheit sollten nun keineswegs aus der Kulturgeschichte des deutschen Volkes gestrichen werden. Ganz konsequent wurden diese Frauen aber immer nur als Einzelerscheinung mit einer Sonderbegabung gesehen. Wenn es irgendwie möglich war, so versuchte man, die Künstlerinnen nachträglich noch in den vorgegebenen ideologischen Rahmen zu pressen. Bei der Würdigung Clara Schumanns z. B. sollte vor allem ihre gute Ehe und die vorbildliche Erfüllung ihrer Mutterpflichten herausgestellt werden, und vor der Komponistin Schumann war die Pianistin zu betonen, ${ }^{6}$ d. h. die eher weibliche Fähigkeit zu interpretieren (nachzuempfinden), nicht die männlich schöpferische Begabung sollte im Mittelpunkt stehen. Obwohl die schöpferisch-künstlerisch tätige Frau sich nach den Vorstellungen der Nationalsozialisten nicht ideologiekonform verhielt, so wurden außerordentliche Begabungen doch nicht übersehen, sondern vielmehr für die Zwecke des Regimes ausgenutzt. Ähnlich verfuhr man auch bei der Behandlung von Spitzensportlerinnen. Bei großen Kulturleistungen, die der Welt das hohe Niveau deutscher Kultur deutlich machten und bei überdurchschnittlichen sportlichen Leistungen, die den deutschen Menschen als außergewöhnlich kennzeichneten, war es den Machthabern ziemlich egal, wer sie hervorgebracht. hatte. Zudem waren die Werke weiblicher Künstler, ja im Grunde allein das Vorhandensein von Künstlerinnen, ein wichtiger „Gegenbeweis“ zu den Behauptungen, die Frau würde im NS-Staat auf den häuslichen Bereich beschränkt. Diese „Beweisführung“ sollte sowohl im Ausland als auch im Inland Zweifel beseitigen - also auch hier zählte nicht die Frau als Persönlichkeit, sondern in der Hauptsache wurde sie als Hilfsorgan des Regimes ausgenutzt.

\section{b) Die Frau als Kunstobjekt}

Das nationalsozialistische Kunstverständnis war durch Definitionen wie die folgenden charakterisiert: „Die neue deutsche Kunst wird eine völkische Gemeinschaftkunst sein. Dafür

zung des Künstlers innerhalb des Systems aufzuzeigen. Nicht das Anliegen des Künstlers ist also Thema, sondern der „Auftrag“ des Regimes an die Kunst.

2 Eggener, S. $25 . \quad{ }^{3}$ Ebenda.

4 Ilse Plen: Die künstlerisch schaffende Frau. In: FW 3 (1934/35), S. 552.

${ }^{5}$ I.v. W. „Das Gewissensgesetz in der Kunst“. In: VB (Süddt. Ausg.) 7. I I . 1934.

${ }^{6}$ Zeitschriften-Dienst Nr. 302. Die Tendenz, Komponistinnen zu ignorieren setzte schon vor 1933 ein und hat sich offenbar bis in die Gegenwart fortgesetzt. Seit etwa zweieinhalb Jahren bemüht sich der in- 
müssen erst die lebendigen Voraussetzungen geschaffen werden. Denn Kunst ist in ihrem Geschehen keine ästhetische Angelegenheit, sondern eine biologische . . Die Gemeinschaft des Blutes, der Rasse, des Volkes ist die alles Leben und alle Kultur erzeugende Macht." ${ }^{7}$ Elf Jahre später hieß es, die Kunst „tritt aus dem Bereich des Nur-Ästhetischen in den AuchPolitischen, sie wird - im besten Sinne - Führungsmittel. “8 Die Zeiten des l'art pour l'art schienen ab I 933 also endgültig vorbei, auch die Kunst hatte in den Dienst des Regimes zu treten. ${ }^{9}$ Die Instrumentalisierung der Kunst für die Zwecke des Systems läßt sich besonders gut in der Malerei nachweisen. Zwei Ansprüche stellten die Nationalsozialisten an die neue Malerei, sie mußte neben körperlicher und charakterlicher Schönheit und Sauberkeit Lebensbejahung und Diesseitigkeit der Lebenserfüllung ausdrücken ${ }^{10}$ und die Darstellung des Menschen war so konkret zu gestalten, daß Mann und Frau rein anatomisch auch ihren „höchsten Aufgaben“ genügen könnten. „In dieser richtig gesehenen und wiedergegebenen Zweckmäßigkeit liegt ein letzter Maßstab für die Schönheit.“"11

Das Bild der Frau in der NS-Kunst war hauptsächlich das der nackten Frau. ${ }^{12}$ „Dieses verstärkte Interesse der heutigen Malerei am Aktbild beweist besonders deutlich den Willen der neuen deutschen Malerei, über die malerisch artistischen und bloß ästhetischen Probleme in das Gebiet einer weltanschaulichen, kulturgeschichtlich dokumentarischen Aussage hinauszugreifen. Das Aktbild hat zu allen Zeiten über das individuell Variable und Zeitgebundene des malerischen Stils besonders klar die weltanschauliche Haltung einer Zeit in ihrer Stellung zum Problem Mensch dokumentiert. Mit anderen Worten, das Aktbild war immer ein besonders deutlicher Ausdruck der sittlichen, soziologischen und religiösen Anschauungen einer Zeit." 13

In der Tat ist an der Aktmalerei, ebenso wie an der Plastik, die Stellung des Künstlers und des ihn fördernden Staates zur Frau überdeutlich abzulesen. Die dargestellten Frauen sind meist jung und im Stadium der Erwartung. Das Ziel dieses Wartens - der Mann - wird zwar, wenn überhaupt, verschlüsselt, oft in mythologischen Szenen ins Bild gebracht, er wird aber dennoch demonstrativ herausgestellt. ${ }^{14}$ Die Frau signalisiert Hingabe und Anpassungsbereitschaft, sie steht dem Mann, der sie „erwählt“, zur Verfügung, sie ist permanent für ihn da. ${ }^{15}$ Die Frau selbst ist nicht Subjekt, sondern Objekt, sie braucht die Beziehung

ternationale Arbeitskreis „Frau und Musik“ um die in Vergessenheit geratenen Musikerinnen. Peter Schönberger: Auf der Spur vergessener Komponistinnen. In: Frankfurter Rundschau 2 I . 6. r980, S. I 8.

7 Wilhelm Rüdiger: Grundlagen deutscher Kunst. In: Nationalsozialistische Monatshefte 4 (r 933 ), S. 469.

8 A. U. Sander: Jugend und Film. Berlin 1944, S. 12.

9 Die Indienstnahme für das Dritte Reich wurde schon rein äußerlich dadurch deutlich, daß das Propagandaministerium den Bereich Kunst übernahm. Georg Hellack: Architektur und bildende Kunst als Mittel nationalsozialistischer Propaganda. In: Publizistik S (I 960), S. 78.

${ }_{10}$ Werner Rittich: Deutsche Kunst der Gegenwart 2. Bd. Malerei und Graphik. Breslau I 943, S. 44.

${ }^{11}$ Kulturtagung der NSDAP. In: Der Kongreß zu Nürnberg vom 5. bis I0.September I934. München 1934, S. 99. Diese Forderungen, die für die Kunst entwickelt wurden, sollten in dem BDM-Werk „Glaube und Schönheit" konkret umgesetzt werden.

${ }^{12}$ Christian Groß, Uwe Großmann: Die Darstellung der Frau. In: Kunst im Dritten Reich. Frankfurt a.M. o. J. ${ }^{3}$, S. 182.

13 Robert Scholz: Das Problem der Aktmalerei. In: Die Kunst im Dritten Reich 1940, S. 292.

14 Groß, Großmann, S. I 84.

15 Ebenda. Georg Bussmann: Plastik. In: Kunst im Dritten Reich, S. I 16. 
zum Mann für die Erfüllung ihrer Rolle. Symptomatisch für das Mann-Frau-Verhältnis in Malerei und Plastik ist das immer wiederkehrende Thema „Urteil des Paris“. Durch die Art der Frauendarstellung konnte sich jeder männliche Betrachter dieser Kunstwerke als kleiner Paris fühlen, der unter sich anbietenden Objekten wählen kann. Das kam einer Sanktionierung und Legitimierung der sexuellen Herrschaft des Mannes gleich, was nicht nur ideologiekonform war, sondern auch als Ersatz für den realen Machtentzug des Mannes in der Öffentlichkeit dienen konnte. Zudem diente die Darstellungsweise natürlich auch der Bevölkerungspolitik, denn die zahlreichen Aktdarstellungen können wohl ohne weiteres als Inspirationshilfe interpretiert werden. Die nächste Stufe von Frauenbildern sind die Mutterdarstellungen. Mutterschaft wurde grundsätzlich als harmonischer, konfliktfreier Zustand geschildert, der Hinweis auf die Natürlichkeit des Mutterdaseins war meist kaum zu übersehen: Zum größten Teil sind Mutter und Kind in freier Natur - z. B. umgeben von wogenden Ähren - abgebildet.

Kritik gegen die degradierende und funktionalisierte Darstellung der Frau wurde bereits von Zeitgenossinnen geübt. Auf die Frage, warum immer nur die Frau unter den diversen ausstellbaren Gegenständen erscheine, folgte die (selbst)kritische Antwort: „Weil der Mann Persönlichkeit der Gesellschaftsordnung ist. Der Frau ist es bis heute nicht gelungen, ihrer Wertung als Gegenstand ein Ende zu machen." ${ }^{16}$ Die Ideologie und die realen Verhältnisse des NS-Regimes erschwerten diesen Prozeß der Umwertung noch zusätzlich.

Die bedeutende Stellung, die die Bevölkerungspolitik in Malerei und Plastik einnahm, ist im Film eigenartigerweise nicht festzustellen. Obwohl der Film als wichtigstes Volksbildungsmittel betrachtet wurde, ${ }^{17}$ spielte er bei der bevölkerungspolitischen Propaganda nur eine geringe Rolle. ${ }^{18}$ Teilweise ergab sich diese Sonderentwicklung wohl aus dem mangelhaften Angebot an talentierten Drehbuchautoren, ${ }^{19}$ die in der Lage waren, das Leben einer kinderreichen Familie als erstrebenswert, aber dennoch glaubwürdig, darzustellen. Eine weitere Erklärung ist vermutlich darin zu sehen, daß der Film insgesamt die Aufgabe hatte, die Flucht aus der Wirklichkeit zu erleichtern, ${ }^{20}$ echte Konflikte und Probleme, wie sie in einer kinderreichen Familie zwangsläufig auftauchen, hätten dieses Ziel entweder sabotiert, oder man hätte sich mit einer unglaubwürdigen Darstellung nur lächerlich gemacht. Aber auch ohne Mutterbild vermittelte der Film noch genügend ideologickonforme Rollenmuster. In eindeutig politischen Filmen war die Zahl der weiblichen Hauptdarsteller signifikant niedrig, hier herrschte ein deutliches Úbergewicht männlicher Schauspieler ${ }^{21}$ - Frauen hatten bei politischen Kämpfen eben nichts zu suchen. Bei den Spielfilmen gab es hauptsächlich zwei Kategorien von Frauen: das süße Mädel, das durch Heirat in der sozialen Rangskala

\footnotetext{
16 R-B.: Rückblick auf die Ausstellung, Die Frau'. In: Die Deutsche Kämpferin (1 933/34), S. 6 r.

17 Hildegard Passow: Die Frau und der Film. In: FW 2 (1933/34), S. 442.

18 Werner-Herbert Rascher: Wünsche, die wir an den Film haben. In: FW 7 (1938/39), S. 53 I beklagt diese Tatsache sehr.

19 Wie sehr gute Drehbuchautoren überhaupt fehlten, zeigt sich bereits daran, daß Erich Kästner trotz seines Schreibverbots mit offizieller Genehmigung das Drehbuch zu einem Film für das 25 . Jubiläum der UFA schrieb. Richard Grunberger: Das zwölfjährige Reich. Wien/München/Zürich I 97 I, S. 404.

20 Ebenda, S. 393.

21 Gerd Albrecht. Nationalsozialistische Filmpolitik. Stuttgart 1969, S. I 46 und S. 20 I .
} 
aufsteigt, und die Luxusfrau, dargestellt als wilde, leidenschaftliche Katze oder als schönes, oberflächliches Weibchen. ${ }^{22}$ Grundsätzlich waren aber beide Frauentypen auf den Mann ausgerichtet.

Betrachtet man die dramatische Produktion im nationalsozialistischen Deutschland im Hinblick auf die Stellung der Frau, „so fällt zunächst der Mangel an Frauengestalten überhaupt und der noch größere Mangel an durchgearbeiteten Charakteren auf". Die Frau ist meist nur dekoratives Anhängsel, ihre psychische Situation interessiert nicht. Ansatzweise entwickelt war der Typus der „herben, tapferen Kameradin“, die aber meist nur als Resonanzboden für die Ideen des Mannes diente. Diese Frauenfigur war weitgehend die einzige Alternative zum nur mütterlichen Typ. ${ }^{23}$

Die Literatur hat insgesamt dazu beigetragen, die Inhalte der NS-Ideologie „denkgerecht“ zu artikulieren und zu verbreiten. ${ }^{24}$ Hauptanliegen der Frauenliteratur war es, die „Verwurzelung in der Familie, Einreihung in das Ganze, auch wenn es schwer ist, Besinnlichkeit und Treue" darzustellen ${ }^{25}$ und als nachahmenswerte Ideale zu propagieren. Aber auch Abenteuer- und Kriegsbücher wurden von BDM-Führerinnen zur Lektüre empfohlen. ${ }^{26}$ Vor allem die Kriegsbücher, die von den Heldentaten der deutschen Armee und vom Heldentum deutscher Soldaten erzählten, sollten Anlaß zum Nacheifern geben, wenn auch in bescheidenerem Rahmen, gleichzeitig sollten sie der Großartigkeit deutscher Tapferkeit Ausdruck verleihen, auf die man nur stolz sein konnte.

Die Tendenz, die Frau für das System zu funktionalisieren, läßt sich also auch im Bereich der Kunst nachweisen, die Frau war im wahrsten Sinne des Wortes Kunstobjekt geworden.

\section{Frau und Unterhaltungsliteratur}

\section{a) Die literarische Produktion und ibre Verbreitung}

Die Fragen, was der Schriftsteller leisten und was sein Werk bewirken solle, wurden im Nationalsozialismus sehr klar und sehr einseitig beantwortet. Der Schriftsteller hatte in erster Linie Erzieher ${ }^{1}$ und politischer Soldat des Führers zu sein. ${ }^{2}$ Als „Dichter im Dienst“ war die Erfüllung seiner Aufgabe ein politischer Auftrag, ${ }^{3}$ und ,.. die Funktion des volkhaften Dichters rückt damit so dicht wie noch nie neben den Verantwortungskreis des Staatsmannes. ${ }^{4}$

${ }^{22}$ Lore Bauer-Hundsdörfer: Dichterinnen sprechen vom Volk zum Volk. In: Jahrbuch der Reichsfrauenführung 1936, S. 345 .

${ }^{23}$ Ilse Pitsch: Das Theater als politisch-publizistisches Führungsmittel im Dritten Reich. Diss. Münster 1952, S. I 60 f.

${ }^{24}$ Klaus Vondung: Völkisch-nationale und national-sozialistische Literaturtheorie. München 1973, S. 7.

${ }^{25}$ Agnes Herkommer: Das Frauenbildnis im neuen deutschen Schrifttum. In: Ellen Semmelroth, Renate von Stieda (Hrg.): N. S. Frauenbuch, München 1934, S. I 84.

${ }^{26}$ Yella Erdmann: Wesensgemäße Mädchenerziehung. In: Die Frau 43 (1935/36), S. 3 1 3. Bücher, die wir uns zu Weihnachten wünschen für den BDM. In: Führerinnendienst BDM, Folge 1, Dezember 1938, S. 3 I.

' Dietrich Strothmann: Nationalsozialistische Literaturpolitik. Bonn i 960, S. 83.

${ }^{2}$ Karl Heinz Hederich: Nationalsozialismus und Buch. Mainz 1937, o.S.

${ }^{3}$ Strothmann, S. 84.

"Heinz Kindermann: Die deutsche Gegenwartsdichtung im Aufbau der Nation. Berlin 1936, S. 10. 
Die Dichtung des volkhaften Dichters ,ist erfüllt vom inbrünstigen Glauben an eine große deutsche Sendung“, sein Menschenbild „.. . strebt auch in der Bauern- und Arbeiterdarstellung, einer ausgesprochenen heldischen Lebensgestaltung zu, für die die Begriffe der Treue, des Opfers, der Verantwortung zu selbstverständlichen Bewährungswerten vor Volk und Rasse werden." ${ }^{5}$

Selbst das Kinderbuch mußte politisch sein und in den Dienst von Volkstum und Rasse gestellt werden. ${ }^{6}$ Dasselbe galt natürlich für das Jugendbuch, es hatte „sich dem zielbewußten Erziehungsprogramm, das Staat und Reichsjugendführung aufgestellt haben, ein- und unterzuordnen. " 7 m die gewünschte Richtung bei der Buchproduktion zu erreichen und abzusichern, entwickelte das System einen gewaltigen Uberwachungs- und Lenkungsapparat, der auch ein Indiz dafür war, daß die literarische Produktion als Einflußmöglichkeit sehr ernst genommen wurde. ${ }^{8}$

Schon vor 1933 begann, von „Säuberungsaktionen“ begleitet, der institutionelle Aufbau des Kontrollsystems. ${ }^{9}$ Schriftsteller mußten dann in sogenannten "Autorenstammrollen“ eingetragen sein, Voraussetzung dafür war die schriftliche Verpflichtung, dem NS-Staat zu dienen. ${ }^{10}$ Das gesamte Bücherei- und Verlagswesen, literarische Gesellschaften und sogar die Buchwerbung wurden zwar von verschiedenen Partei- und Staatsstellen überwacht, ${ }^{11}$ aber dadurch, daß sowohl der private Buchhandel als auch das private Bücherei- und Verlagssystem bestehen blieben, unterschied sich die Propaganda von der Literaturkontrolle in der UdSSR. Daß die Zensur nur vorverlagert war, fand natürlich keine Erwähnung, wenn auf die Kulturfreiheit verwiesen wurde. ${ }^{12}$

Selbst die Literaturkritik fiel unter die Überwachung des Staates, denn auch die Buchbesprechung hatte eine politisch-weltanschauliche Aufgabe zu erfüllen. Da es sich bei der Rezension um eine so wichtige Aufgabe handelte, daß sie nach dem Verständnis der Nationalsozialisten eine gewisse Lebensreife voraussetzte, durfte der seit 1936 offiziell so genannte Kunstbetrachter erst vom 30 . Lebensjahr an gutachten. ${ }^{13}$ Diese Bestimmung war allerdings nur eine Farce, denn durch staatlich festgelegte Fristen, innerhalb derer ein Buch besprochen sein mußte, blieb für eine echte Auseinandersetzung mit dem Schrifttum ohnehin wenig Zeit. ${ }^{14}$

Den Machthabern reichte es aber nicht, nur das Unerwünschte von Produktion und Verbreitung auszuschließen, in ihren Augen war es auch notwendig, das Lesenswerte zu fördern. Das wichtigste Mittel zur Förderung bestimmter Lesestoffe und Autoren stellten die Büchereien dar. Besonders die Jugendlichen sollten mit Hilfe des Büchereiwesens vor

\footnotetext{
5 Ebenda, S. 8 f.

6 Hugo Wippler: Kleinkind und Bilderbuch. In: Jugendschriften-Warte 4I (1936), S. 29-33.

7 Dora Bessel-Hagen: Grundsätzliches zur Jugendschriftenfrage. In: Nationalsozialistische Mädchenerziehung 1937, S. I 38.

${ }^{8}$ Gleichzeitig spricht diese Aufblähung auch eine deutliche Sprache in bezug auf die Postenhascherei der Pgs. und den herrschenden Kompetenzenwirrwarr.

9 Vgl. dazu Strothmann, S. I6.

10 Ebenda, S. 29.

11 Ebenda, S. 22.

12 Ebenda, S.63. ${ }^{13}$ Ebenda, S. 277.

14 Ebenda, S. 289.
} 
„Schmutz- und Schundliteratur“ bewahrt werden. Vorrangig betrieb man den Ausbau und die Überprüfung von Schülerbüchereien. ${ }^{15}$ Werke, die der weltanschaulich-politischen Grundhaltung widersprachen, waren rücksichtslos aus den Büchereien auszuscheiden, ${ }^{16}$ die Neuanschaffungen hatten nach einer Grundliste zu erfolgen, ${ }^{17}$ die ab 1934 durch regelmäBige Listen erweitert wurde. ${ }^{18}$ Auch die Volksbüchereien steuerte man durch amtliche Literaturverzeichnisse. ${ }^{19}$ Das, was von staatlicher Seite an Literatur erwünscht war, konnte über diese Listen so eindeutig artikuliert werden, daß es an vielen. Orten gelang, beim Neuaufbau einer Volksbücherei einen genormten, einheitlichen Buchbestand einzusetzen. ${ }^{20}$ Trotz dieser Organisierung erwies es sich jedoch als schwierig, die richtigen Themen der Frau nahezubringen. So wurde $x 936$ noch bedauernd festgestellt, daß sich die Jugend nur allmählich von den alten Vorlieben (wie z. B. Karl May) trennte, um sich zeitbezogenen Themen zuzuwenden. ${ }^{21}$ Daß sich die Frau bevorzugt mit Romanen beschäftigte, die Liebe, Ehe und Familie behandelten, wurde zwar geduldet, ${ }^{22}$ gleichzeitig wies man die Frauen aber darauf hin, daß auch sie sich mehr auf Volk und Staat auszurichten hätten. ${ }^{23}$

\section{b) Frauenzeitschriften}

Wie die Literatur, so wurde „die gesamte deutsche Presse . . e einzig und allein in den Dienst der Volksgemeinschaft und des von ihr gewollten Staates gestellt . . Aus der privaten Sphäre des jeweils erreichten Leserkreises wurde die Zeitung erhoben zu einem Organ des vom Volk getragenen Staates. Mit höchsten Aufgaben erhielt sie die höchste Würde: Mittler zu sein zwischen Führer und Gefolgschaft." ${ }^{24}$ Diese Aufgabe der Presse bezog sich auf alle Sparten, d.h. auch die Frauenzeitschriften und Frauenbeilagen hatten Vermittler der Ideologie zu sein. Die Frauenzeitschriften, von ihrer Entstehungsgeschichte her eigentlich eng verbunden mit der Emanzipation der Frau, ${ }^{25}$ wurden im NS-Staat gezielt dazu genutzt, „unpolitische Frauen“ anzusprechen und für das System nutzbar zu machen. Die Zeitschriften, die für dieses Vorhaben nicht geeignet waren, wie z. B. Die Deutsche Kämpferin, fielen entweder einem Verbot zum Opfer ${ }^{26}$ oder mußten ihr Erscheinen während des Krieges ,aus Papiermangel“ einstellen.

Die grundsätzliche Bedeutung der Ideologievermittlung durch spezielle Frauenzeitschriften und Beilagen zeigte sich an zwei Tatsachen: an der Existenz einer „parteiamtlichen Frauenzeitschrift" (NS Frauenwarte), die nicht nur für Parteigenossinnen gedacht war, und daran,

15 Nach Strothmann, S. 34 Anm. 39 entstanden allein zwischen 1937 und r 94 I noch 55000 neue Schülerbüchereien.

${ }^{16}$ Reichswaltung der NSLB (Hrsg.): Die Schülerbücherei. Leipzig o. J., [1 939], S. 23-27.

17 Ebenda, S. I 13.

18 Zentralblatt für die gesamte Unterrichtsverwaltung in Preußen I 933, S. 306-308.

19 Strothmann, S. 34. ${ }^{20}$ Ebenda, S. I 45.

${ }^{21}$ Herbert Beck: Forderung und Wirklichkeit im Jugendbuchverlag. In: Börsenblatt für den Deutschen Buchhandel. Leipzig 1936, S. I 2. 1936, Nr. 283 , S. 1063.

${ }^{22}$ Nach Strothmann, S. I 6 I f. wurde das Lesen leichter Liebesromane zwar nicht gern gesehen, aber hingenommen, Courths-Mahler allerdings wurde nicht mehr geduldet.

${ }^{23}$ Hans Hofmann: Frau und Volksbücherei. In: FW 6 (1937/38), S. I 66.

${ }^{24}$ Friedhelm Kaiser: Die deutsche Zeitung. Münster/Westf. I939, S. 2 r f.

2s Vgl. dazu Josefine Trampler-Steiner: Die Frau als Publizistin und Leserin. Diss. München 1938, S. 9-64.

${ }^{26}$ Nach Trampler-Steiner, S. I 28-1 32 mußten bis I 93876 Zeitschriften ihr Erscheinen einstellen. 
daß 1937 bereits $48,38 \%$ der NS-Presse eine Frauenbeilage hatte. ${ }^{27}$ Sowohl die NS Frauenwarte als auch die Frauenbeilage des Völkischen Beobachters, die als typisch gelten können, unterschieden sich auf der Anschauungsebene nicht wesentlich von den heute üblichen Frauenzeitschriften. ${ }^{28}$ Mode, Rezepte, Ehe- und Familienprobleme waren die Hauptthemen, Politisches wurde nur in verschlüsselter Form vermittelt. Bereits die Auswahl der Themen drängte die Frau in den Bereich Hausfrau und Mutter, die inhaltliche Aufbereitung der oben angesprochenen Gegenstände verstärkte diese Tendenz. Allein durch diese permanente Rollenfixierung wirkten die Frauenzeitschriften natürlich schon in eine bestimmte politische Richtung. Die inhaltliche Ausgestaltung dieser Rolle war allerdings anders als heute. Die bekannten Ideale, die verständnisvolle Ehefrau, die aufopfernde Mutter, die ihre Kinder für den Staat und nach seinen Forderungen erzieht, die zum Wohl der Volksgemeinschaft auf persönliches Glück verzichtet, wurden in allen Varianten beschrieben und als das erstrebenswerte Frauenlos dargestellt.

Die Einengung der Frau auf ein Hausfrauen- und Mutterdasein wurde (wie heute) durch die Werbung kräftig unterstützt. Graue Wäsche, rauhe Hände und ein wenig attraktives Äußeres waren offensichtlich auch im Dritten Reich zentrale Probleme, falls man der Werbung Glauben schenkte. ${ }^{29}$ Der Grund für den Wunsch nach einem makellosen Aussehen, strahlend weißer Wäsche und ähnlichen Dingen hing damals (wie heute) offensichtlich mit dem „natürlichen Bedürfnis“ der Frau zusammen, zunächst Ehefrau, dann aber auch eine gute Hausfrau und Mutter zu werden. Vertraute man sich den einschlägigen Industrieprodukten an, so schien es, waren die Hauptprobleme des weiblichen Daseins gelöst. Diese Illusion wurde in den Textbeiträgen keineswegs aufgehoben. Existentielle Probleme waren der Frau im NS-Staat scheinbar fremd.

Durch die frauenspezifischen Themen wurden aber zusätzliche, politisch genauso wichtige Ziele unterschwellig verfolgt. Die Modebeilagen verwiesen die Frauen auf bestimmte, im eigenen Land hergestellte Stoffarten und auf Modelle deutscher Modeschöpfer, d.h. sie waren im Grunde kleine Beiträge zur Autarkiepolitik. ${ }^{30}$ Selbst die Sparte Kochrezepte waren diesem Aspekt untergeordnet. Als Teil der Verbrauchslenkung wurden bestimmte Produkte, je nach Jahreszeit, angepriesen und der Hausfrau wurde bei deren Zubereitung Hilfestellung geleistet.

Weltpolitisch relevante Themen behandelten die Frauenzeitschriften zwar auch, meist waren sie aber für die Frauen besonders „aufbereitet“. Beliebt war vor allem die Schilderung der „Frauennot“ in bestimmten Gebieten, z. B. im Saarland oder der „Ostmark“. Offensichtlich sollte die deutsche Frau über die emotionale Solidarisierung mit den „rassenverwandten“"Geschlechtsgenossinnen zu einer positiven Einschätzung der staatlichen Maßnahmen gelangen. Eine rationale Auseinandersetzung mit Fragen der Außenpolitik war dagegen weder erwünscht noch notwendig.

\footnotetext{
${ }^{27}$ Philipp-Bouhler: Adolf Hitler. Lübeck 1932, (4.-6. Tsd.), S. 47.

${ }^{28}$ Gemeint sind hier nicht die feministischen Zeitschriften wie z. B. Emma, Courage etc.!

${ }^{29} \mathrm{FW}$ passim.

${ }^{30}$ Die Bedeutung, die diesem Problem zugemessen wurde, läßt sich an der Errichtung des Deutschen Modeamtes ablesen, das in Konkurrenz und Abgrenzung zur Pariser Haute Couture gegründet wurde. Vgl.: Wettkämpfe deutscher Mode. In: Vossische Zeitung 6. 7. 1933.
} 